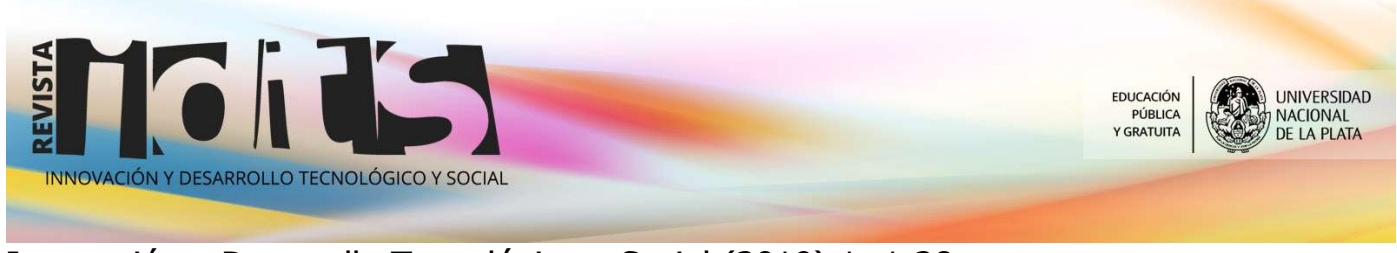

Innovación y Desarrollo Tecnológico y Social (2019) 1: 1-28

\title{
Eco-turbina. Turbo ventilador eléctrico 220 (VAC) - 50 (Hz), de bajo consumo: eficiente energéticamente.
}

\author{
Anderson, Ibar Federico ${ }^{123}$ \\ ${ }^{1}$ Investigador Categoría 3, Secretaría Ciencia y Técnica, Departamento de Diseño Industrial - \\ Universidad Nacional de La Plata. \\ 2Profesor de Educación Tecnológica, Escuela Técnica n² 2 "Independencia", Concordia, Entre \\ Ríos. ${ }^{3} E$-mail: ibar.federico.anderson@gmail.com
}

Resumen Según el World Energy Outlook $2017^{1}$ publicado por la Agencia Internacional de la Energía (AIE) se evidencian algunas tendencias en el sistema energético mundial, en donde los motores eléctricos representarán un tercio del aumento de la demanda de energía eléctrica. Este aumento significa que millones de hogares agregarán electrodomésticos y sistemas de refrigeración. Recientemente la Agencia publicó un muy completo estudio ${ }^{3}$ sobre la situación del uso de aires acondicionados ${ }^{4}$ en el mundo. En la República Argentina, informes anuales de CAMMESA5: 2007 y 2016 indican que en ese período hubo un aumento del 45\% en el consumo eléctrico en todos los sectores, lo que significa un problema en la generación y transmisión de energía. Se tornó una necesidad todas las medidas que se puedan tomar en sentido de la Eficiencia Energética (EE); lo que por otro lado significa una oportunidad en el diseño y desarrollo de productos industriales más eficientes en el consumo de la energía eléctrica.

En clara orientación con esta línea ética de reducción de la huella de carbono ${ }^{6}$ y sustentada científicamente en el impacto ambiental, se desarrolló una turbina eléctrica de 220 (voltios), $50(\mathrm{~Hz})$ de corriente alterna (AC), para ser aplicado a motores de ventiladores'. Reduce un $59 \%$ el consumo de energía eléctrica, medida en kWh (kilo-Watts-hora), que es el modo en que se factura el consumo de energía. Según la Norma IRAM 62480:2017 se obtuvo una EE Tipo: A. Con un consumo de energía inferior a $55 \%$ del valor nominal; lo que representa $15 \mathrm{kWh} / \mathrm{mes}$, valor que se calcula durante una (1) hora por día a máxima potencia (25 vatios para el prototipo).

Cabe destacar que existen normas $I E^{8}$ de eficiencia energética para motores eléctricos, que no se pudieron constatar, dado que exceden a los recursos disponibles para este trabajo.

1 International Energy Agency. World Energy Outlook 2017 [On line]. Available: https://www.iea.org/weo2017/ [Accessed: 25-jan-2019]

${ }^{2}$ International Energy Agency. Energy Efficiency. The global exchange for energy efficiency policies, data and analysis [On line]. Available: https://www.iea.org/topics/energyefficiency [Accessed: 25-jan-2019]

${ }^{3}$ International Energy Agency. The Future of Cooling. Opportunities for energy-efficient air conditioning [On line]. Available: https://webstore.iea.org/the-future-of-cooling [Accessed: 25-jan-2019]

${ }^{4} \mathrm{El}$ uso de aires acondicionados y ventiladores eléctricos para mantenerse fresco representa casi el $20 \%$ del total de la electricidad utilizada en los edificios de todo el mundo en la actualidad.

${ }^{5}$ CAMMESA, Compañía Administradora del Mercado Mayorista eléctrico [<en línea]. Disponible en: http://portalweb.cammesa.com/default.aspx [Accedido: 25-ene-2019]

${ }^{6}$ La huella de carbono se conoce como gases de efecto invernadero (GEI) emitidos por efecto directo o indirecto de un individuo, organización, evento o producto. Tal impacto ambiental es medido llevando a cabo un inventario de emisiones de GEI o un análisis de ciclo de vida (ACV), siguiendo normativas internacionales reconocidas, tales como ISO 14064, PAS 2050 o GHG Protocol entre otras.

7Los motores consumen el 46\% del total mundial de electricidad (Fuente IEA: Efficiency Series, 2011).

${ }^{8}$ La norma IEC 60034-30-1 es la que establece el Código IE de eficiencia en motores eléctricos. 
Innovación y Desarrollo Tecnológico y Social (2019) 1: 1-28

El objetivo ha consistido en aproximarnos de un modo más simple (tecnología) y económico (costos) a los variadores de frecuencia (VDF) o drivers, que son una tecnología que reduce la energía eléctrica, manteniendo constante la relación tensión/frecuencia (volts/hertz) con una electrónica compleja y costosa (como los transistores bipolares de puerta aislada: IGBTs). Aquí se ha resuelto el problema manteniendo no-constante la relación $(\mathrm{V} / \mathrm{Hz})$ con un Triac BT 137 para uso en motores de inducción monofásicos de $220(\mathrm{~V}), 50(\mathrm{~Hz})$ de corriente alterna (AC).

Para construir esta tecnología menos costosa (económicamente) y menos compleja (electrónicamente), se analizó la existencia -probada en el mercado-de otras aplicaciones tecnológicas similares, que puedan ser adaptadas y ensambladas a otras tecnologías también existentes; y que este ensamble pueda ser realizado de modo barato y funcional.

Este control de potencia para motores a-sincrónicos fue utilizado en un motor sincrónico de tipo PMSM. Las actividades llevadas a cabo para la construcción del prototipo son: adoptar un motor sincrónico de tipo PMSM (con rotor de imanes permanentes de ferrite) obtenido a partir del estator de una electrobomba de lavavajillas de 65 (watts) de potencia, acoplado a las paletas de un rotor de un motor a-sincrónico de espiras de sombra ${ }^{10}$ de microondas; que se controla mecatrónicamente con un control de potencia de disparo por Triac BT137 atenuador de onda de tensión (Voltios) e intensidad de la corriente (Amperios).

\section{Palabras claves: Energía; Electricidad; Eficiencia energética; Motor; Turbina; Ventilador}

Recibido: 10/12/2018 Aceptado: 26/04/2019

DOI: https://doi.org/10.24215/26838559e001

\footnotetext{
${ }^{9}$ La IEC $61800-9-2$ se focaliza en la interacción de motores con los variadores de frecuencia (VFD). El 40\% y el $60 \%$ de todos los sistemas de motores se beneficiarían del uso adecuado de los drivers.

${ }^{10}$ Espira de defrager o espira de arranque (espira en cortocircuito).
} 


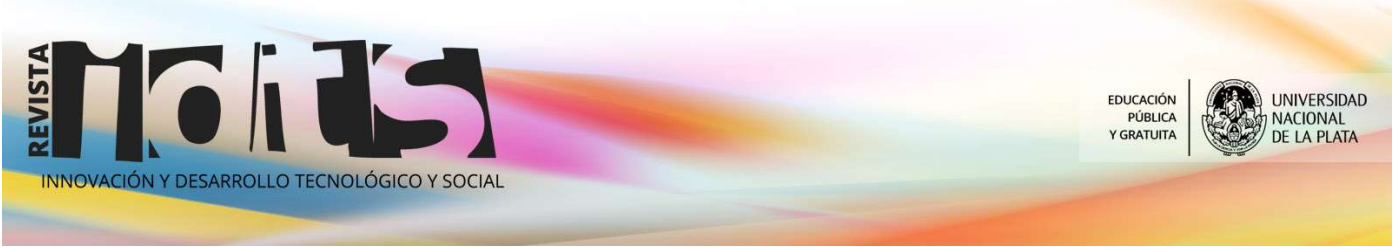

Innovación y Desarrollo Tecnológico y Social (2019) 1: 1-28

\title{
Eco-turbine.Turbo electric fan 220 (VAC) - 50 (Hz), low consumption: energy efficient.
}

\begin{abstract}
According to the World Energy Outlook 2017 published by the International Energy Agency (AIE), some trends in the global energy system are evident, where electric motors will represent a third of the increase in the demand for electric power. This increase means that millions of homes will add appliances and refrigeration systems. Recently the Agency published a very comprehensive study on the situation of the use of air conditioners in the world. In the Argentine Republic, annual reports of CAMMESA: 2007 and 2016 indicate that in that period there was a $45 \%$ increase in electricity consumption in all sectors, which means a problem in the generation and transmission of energy. It became a necessity all the measures that can be taken in the sense of Energy Efficiency (EE); what on the other hand means an opportunity in the design and development of industrial products more efficient in the consumption of electric power.

In a clear orientation with this ethical line of reduction of the carbon footprint and supported scientifically in the environmental impact, an electric turbine of 220 (volts), $50(\mathrm{~Hz})$ of alternating current (AC) was developed to be applied to motors of fans.It reduces the consumption of electrical energy by 59\%, measured in $\mathrm{kWh}$ (kilo-Watts-hour), which is the way in which energy consumption is invoiced. According to IRAM Standard 62480: 2017, an EE Type was obtained: A. With an energy consumption lower than 55\% of the nominal value; which represents $15 \mathrm{kWh} /$ month, value that is calculated during one (1) hour per day at maximum power (25 watts for the prototype).

It should be noted that there are IE energy efficiency standards for electric motors, which could not be verified, given that they exceed the resources available for this work. The tested prototype, The objective has been to approximate in a simpler (technology) and economic (cost) to the frequency variators (VDF) or drivers, which are a technology that reduces electrical energy, keeping constant the voltage / frequency ratio (volts / hertz) with complex and expensive electronics (such as insulated gate bipolar transistors: IGBTS). Here the problem has been solved by keeping the ratio $(\mathrm{V} / \mathrm{Hz})$ non-constant with a Triac $B T 137$ for use in single-phase induction motors of $220(\mathrm{~V}), 50(\mathrm{~Hz})$ alternating current $(\mathrm{AC})$.

In order to build this technology that is less expensive (economically) and less complex (electronically), the existence - proven in the market - of other similar technological applications, which can be adapted and assembled to other existing technologies, was analyzed; and that this assembly can be carried out cheaply and functionally.

This power control for a-synchronous motors was used in a PMSM type synchronous motor. The activities carried out for the construction of the prototype are: adopt a synchronous motor of PMSM type (with rotor of ferrite permanent magnets) obtained from the stator of a dishwasher electric pump of 65 (watts) of power, coupled to the pallets of a rotor of an a-synchronous motor of microwave shadow turns; which is controlled mechatronically with a Triac power control by Triac BT137 voltage wave attenuator (Volts) and current intensity (Amperes).
\end{abstract}

Key words: Energy; Electricity; Energy efficiency; Engine; Turbine; Fan 


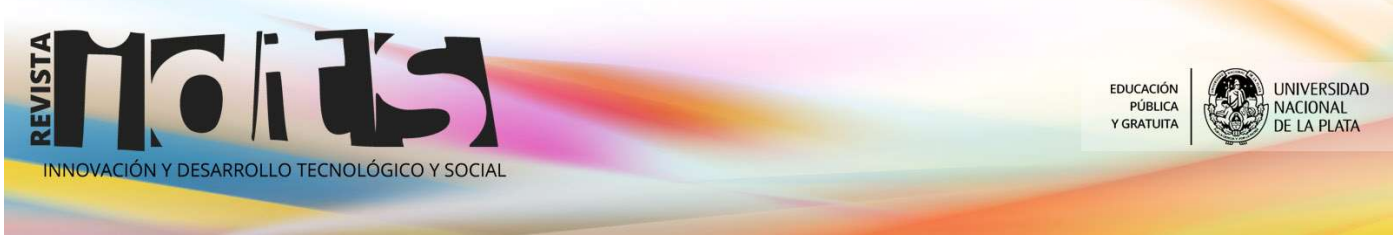

Innovación y Desarrollo Tecnológico y Social (2019) 1: 1-28

\section{Novedad u originalidad local en el conocimiento}

Construir esta tecnología menos costosa (económicamente) y menos compleja (electrónicamente) consistió en utilizar otras tecnologías existentes y recombinarlas de un modo nuevo de modo tal que se respete la definición de novedad de la Ley de patentes de inventos (Ley 24481) ${ }^{11}$, y modelos de utilidad.

El Artículo 4, inciso D de la Ley sostiene:

d) Habrá actividad inventiva cuando el proceso creativo o sus resultados no se deduzcan del estado de la técnica en forma evidente para una persona normalmente versada en la materia técnica correspondiente. (Ley 24481, Artículo $4^{\circ}{ }^{\circ}$ Inc. D).

Efectivamente, la novedad radica en que la abundante bibliografía de electrotecnia y máquinas eléctricas, dice que los denominados circuitos de control de la velocidad de motores eléctricos de inducción a-sincrónicos (asíncronos) de corriente alterna (AC) de media y baja potencia, se realizan por dispositivos electrónicos desde una potencia nominal de 300 (W) hasta 5000 (W). Los motores de inducción (asíncronos) que pueden ser controlados corresponden a los del tipo desarrollado en la patente $\mathrm{n}^{\circ} 381.968$ de Nikola Tesla ${ }^{12}$, del 1 de mayo de 1888.

Nunca hacen referencia a que dichos circuitos electrónicos de control de potencia por materiales semiconductores bidireccionales (Triac) pueden ser aplicados a los motores síncronos (sincrónicos) de tipo $\mathrm{PMSM}^{13}$; ya sean de imanes de ferrite de 4000 (Gauss) o de tierras raras de neodimio $\left(\mathrm{Nd}_{2} \mathrm{Fe}_{14} \mathrm{~B}\right)$ de gran intensidad de campo magnético: entre 12 y 14 mil Gauss (1,2-1,4 Tesla).

\section{Grado de relevancia}

El Ministerio de Energía de la Nación ${ }^{14}$ en la República Argentina, posee una Subsecretaría de Ahorro y Eficiencia Energética $(E E)^{15}$ creada por el Decreto Presidencial

\footnotetext{
${ }^{11}$ Ministerio de Justicia y Derechos Humanos de la república Argentina. Administración Pública nacional. Decreto 231/2015. Modificación del Decreto 357/2002. Buenos Aires [En línea]. Disponible en: http://servicios.infoleg.gob.ar/infolegInternet/anexos/255000-259999/257246/norma.htm [Accedido: 25ene-2019]

${ }^{12 N i k o l a ~ T e s l a ~(1987) . ~}$

13PMSM: PermanentMagnetSynchronous Motor (traducido como: motor síncrono de imán permanente).

14 Secretaría de Energía del Gobierno de la República Argentina. [En línea]. Disponible en: https://www.argentina.gob.ar/energia [Accedido: 25-ene-2019]

${ }^{15}$ Secretaría de Energía del Gobierno de la República Argentina. Ahorro y eficiencia energética [En línea]. Disponible en: https://www.argentina.gob.ar/energia/ahorro-y-eficiencia-energetica [Accedido: 25-ene2019]
} 


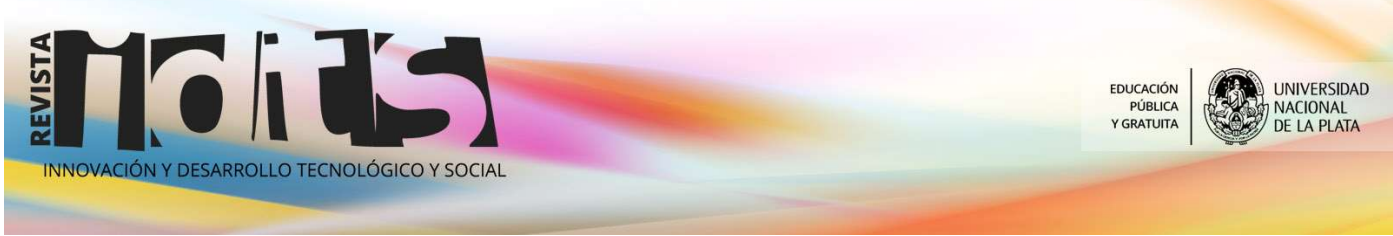

Innovación y Desarrollo Tecnológico y Social (2019) 1: 1-28

$231 / 15^{16}$, lo cual indica la relevancia política y estratégica para el país que posee la $\mathrm{EE}^{17}$ para reducir el consumo de energía eléctrica. Esto se traduce en políticas ${ }^{18}$ para la utilización inteligente ${ }^{19} y$ responsable de la energía en diversas áreas como: educación, sectores productivos, edificación y sector público, transporte, etcétera.

La importancia política y estratégica nacional se manifiesta en un portal de $\mathrm{EE}^{20}$ en la web del gobierno dondese encuentra la etiqueta ${ }^{21}$ de EE, Norma IRAM ${ }^{22}$ 62480:2017, con consejos útiles tanto para el uso responsable (ahorro o reducción del consumo) de la energía y el uso eficiente (optimizar el empleo de la energía, utilizando la misma o menos energía). Dicho de otra manera, la EEsignifica producirigual o más con menos energía.

Un apartado especial con una guía de EE para motores eléctricos ${ }^{23}$, lo que ahorra dinero e incrementa competitividad.

Con un claro objetivo de incorporar la EE en la educación formal en los tres niveles obligatorios (escuela primaria, secundaria y terciaria). Promover convenios y acuerdos con universidades, cámaras empresarias, organizaciones de la sociedad civil y todas aquellas instituciones cuyo objetivo sea mejorar la EE.

\section{Grado de pertinencia:}

\footnotetext{
${ }^{16}$ Ley 24481, República Argentina. Ministerio de Justicia y Derechos Humanos de la Nación. Presidencia de la Nación. InfoLEG: Información Legislativa [En línea]. Disponible en: http://servicios.infoleg.gob.ar/infolegInternet/anexos/25000-29999/27289/norma.htm [Accedido: 25-ene2019]

${ }^{17}$ La Eficiencia Energética es una práctica/forma de gestionar el crecimiento de la energía, obteniendo un resultado igual con menor consumo ó un resultado mayor consumiendo lo mismo. (Fuente: IEA http://www.iea.org/topics/energyefficiency)

${ }^{18}$ Secretaría de Energía del Gobierno de la República Argentina. Eficiencia Energética [En línea]. Disponible en: $\quad$ https://www.argentina.gob.ar/energia/ahorro-y-eficiencia-energetica/politica/eficiencia-energetica [Accedido: 25-ene-2019]

${ }^{19}$ Secretaría de Energía del Gobierno de la República Argentina. Usemos nuestra energía de manera inteligente [En línea]. Disponible en: https://www.argentina.gob.ar/energia/uso-inteligente [Accedido: 25ene-2019]

${ }^{20}$ Secretaría de Energía del Gobierno de la República Argentina. Eficiencia Energética. Usemos nuestra energía de manera inteligente [En línea]. Disponible en: https://www.argentina.gob.ar/energia/ahorro-yeficiencia-energetica/eficiencia-energetica [Accedido: 25-ene-2019]

${ }^{21}$ Secretaría de Energía del Gobierno de la república Argentina. Etiqueta de Eficiencia Energética [En línea]. Disponible en: https://www.argentina.gob.ar/energia/eficiencia-energetica/etiqueta [Accedido: 25-ene2019]

${ }^{22}$ Instituto Argentino de Normalización y Certificación, Nuevas Normas IRAM de etiquetado de eficiencia energética, [En 62480:2017 línea]. Disponible http://aplicaciones.iram.org.ar/userfiles/files/medios/10-07/electrosector.pdf [Accedido: 25-ene-2019]

${ }^{23}$ Ministerio de Energía y Minería, Presidencia de la Nación. Subsecretaría de Ahorro y Eficiencia Energética [En línea]. Disponible en: https://www.argentina.gob.ar/sites/default/files/guia de eficiencia energetica para motores electricos.p df [Accedido: 25-ene-2019]
} 


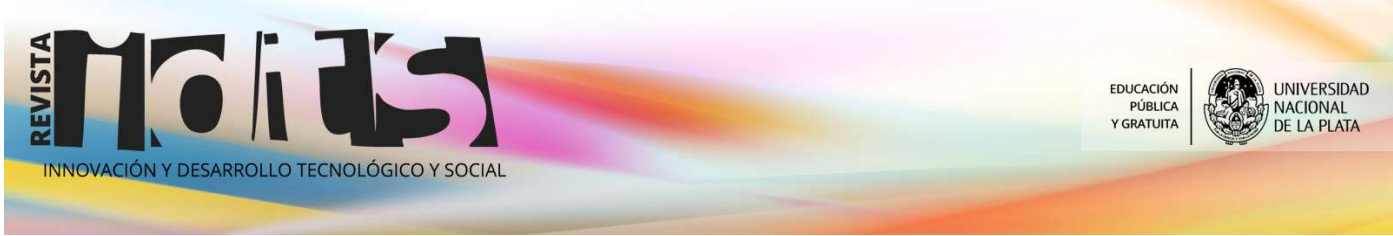

Innovación y Desarrollo Tecnológico y Social (2019) 1: 1-28

Medimos la pertinencia de la innovación tecnológica en el nivel local a partir del catálogo para el Concurso Nacional de innovación tecnológica: INNOVAR ${ }^{24}$. Dependiente del ex Ministerio de Ciencia y Tecnología de la Nación (MINCYT).

En el año 2017 el proyecto fue seleccionado para la Expo Tecnópolis 2017 del MINCYT y quedó registrado en el catálogo digital ${ }^{25}$ y en formato papel. En dicho catálogo no explicitan planos y otra información del diseño del proyecto, que si se incluyen en esta publicación.

Igualmente esta publicación como artículo de divulgación tecnológica, lo que busca o pretende, es que laboratorios de investigación científica y desarrollo tecnológico (I+D), públicos (universidades) y de empresas privadas de la pequeña y mediana empresa (PyMEs); puedan continuar con su desarrollo, mejorarlo y profundizar el desarrollo del producto. También -eventualmente- concluir en el Instituto Nacional de Propiedad Industrial (INPI) ${ }^{26}$, en una patente de modelo de utilidad ${ }^{27}$.

Si este producto, hipotéticamente fuera desarrollado por inversionistas privados $\mathrm{y} / \mathrm{o}$ empresarios (a partir de estudios de mercado más profundos, que no son el objetivo de esta publicación); sería capaz de motorizar la economía, en el sentido de la incorporación de valor agregado en forma de trabajo (intelectual y de mano de obra), de un modo ecológicamente responsable con el medio ambiente, acorde a la Norma ISO $14000^{28}, 14001 / 17$.

\section{Grado de demanda:}

El grado de demanda está establecido en la web del prototipo del producto, donde se presentan la publicaciones de los medios de comunicación masivos: periódicos (en soporte papel y digitales), televisión, internet, links a páginas web o sitios que publican contenido de interés como: canales de radios y Youtube (que alojan contenidos de interés público brindado por universidades)

\footnotetext{
${ }^{24}$ Ministerio de Educación, Cultura, Ciencia y Tecnología de la Nación. República Argentina [En línea]. Disponible en: https://mia.gob.ar/convocatorias/innovar [Accedido: 25-ene-2019]

${ }^{25}$ Catálogo digital, Concurso Nacional INNOVAR 2017. Ministerio de Ciencia, Tecnología e Innovación Productiva de la Nación. República Argentina [En línea]. Disponible en: https://mia.gob.ar/uploads/innovate/catalogo innovar 2017.pd [Accedido: 25-ene-2019]

${ }^{26}$ Instituto Nacional de Propiedad Industrial [En línea]. Disponible en: http://www.inpi.gob.ar [Accedido: 25ene-2019]

${ }^{27}$ Instituto Nacional de Propiedad Industrial [En línea]. Disponible en: http://www.inpi.gob.ar/patentes [Accedido: 25-ene-2019]

28 Sistema de gestión ambiental, ISO 14000 [En línea]. Disponible en: https://www.agroindustria.gob.ar/sitio/areas/d recursos humanos/concurso/normativa/ archivos/000007 Otras $\% 20$ normativas\%20especificas/000000 SISTEMA\%20DE\%20GESTI\%C3\%93N\%20\%20AMBIENTAL \%20ISO\%201400.pdf [Accedido: 25-ene-2019]
} 
Innovación y Desarrollo Tecnológico y Social (2019) 1: 1-28

Nos han realizado entrevistas, reportajes por los medios de soporte papel (diarios), en la ciudad de Concordia y Paraná, adjuntamos figuras(imágenes) y links correspondiente a cada caso.

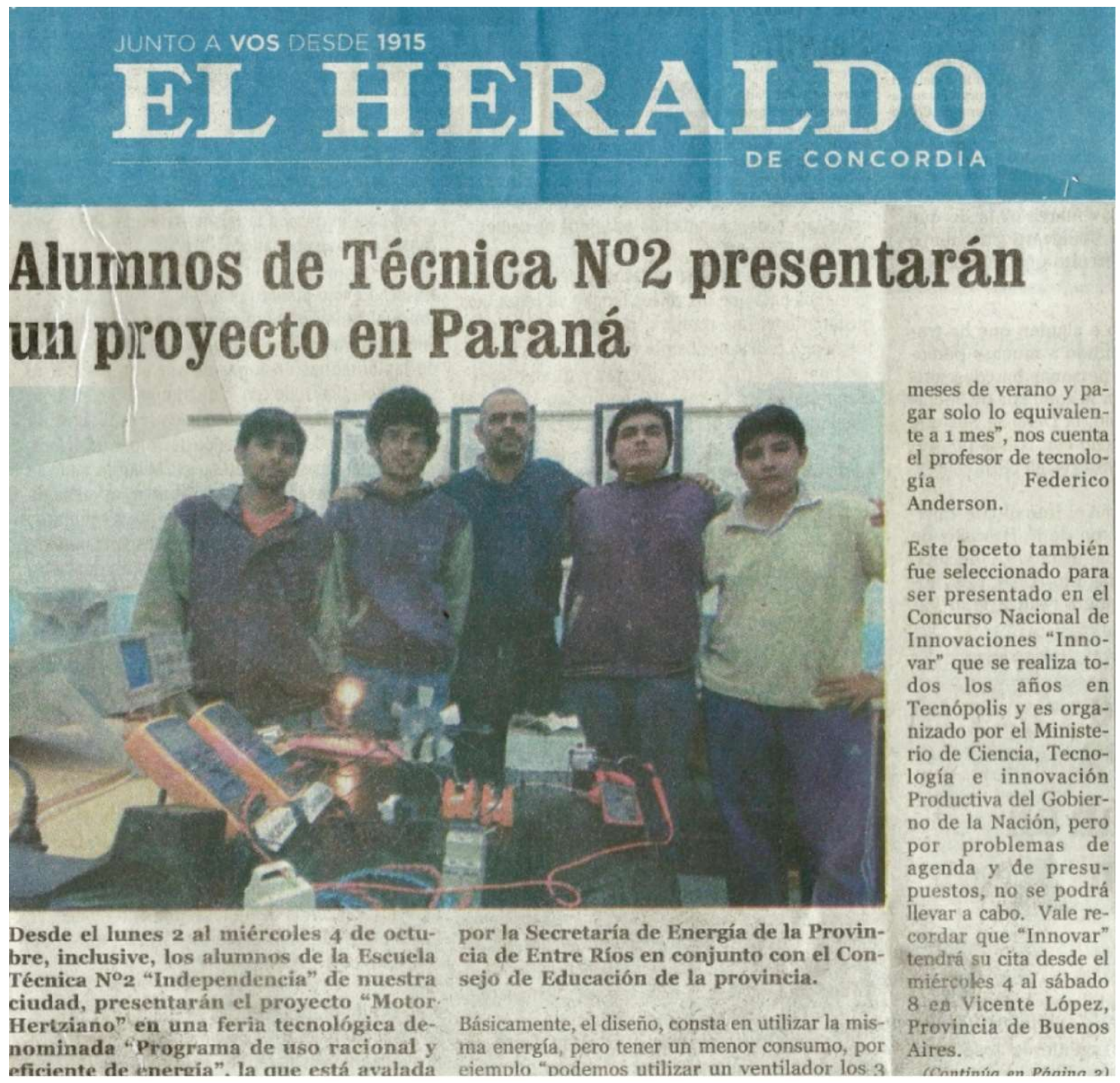

Figura 1. Reportaje periodístico diario "El Heraldo" (Concordia, 30/09/2017), link del reportaje/entrevista: http://www.elheraldo.com.ar/noticias/153057 alumnos-de-tecnica-n2-presentaran-un-proyecto-enparana.htmI /Figure 1.Daily newspaper report "El Heraldo" (Concordia, 30/09/2017), link of the report / interview:http://www.elheraldo.com.ar/noticias/153057 alumnos-de-tecnica-n2-presentaran- a-project-inparana.html 
Innovación y Desarrollo Tecnológico y Social (2019) 1: 1-28

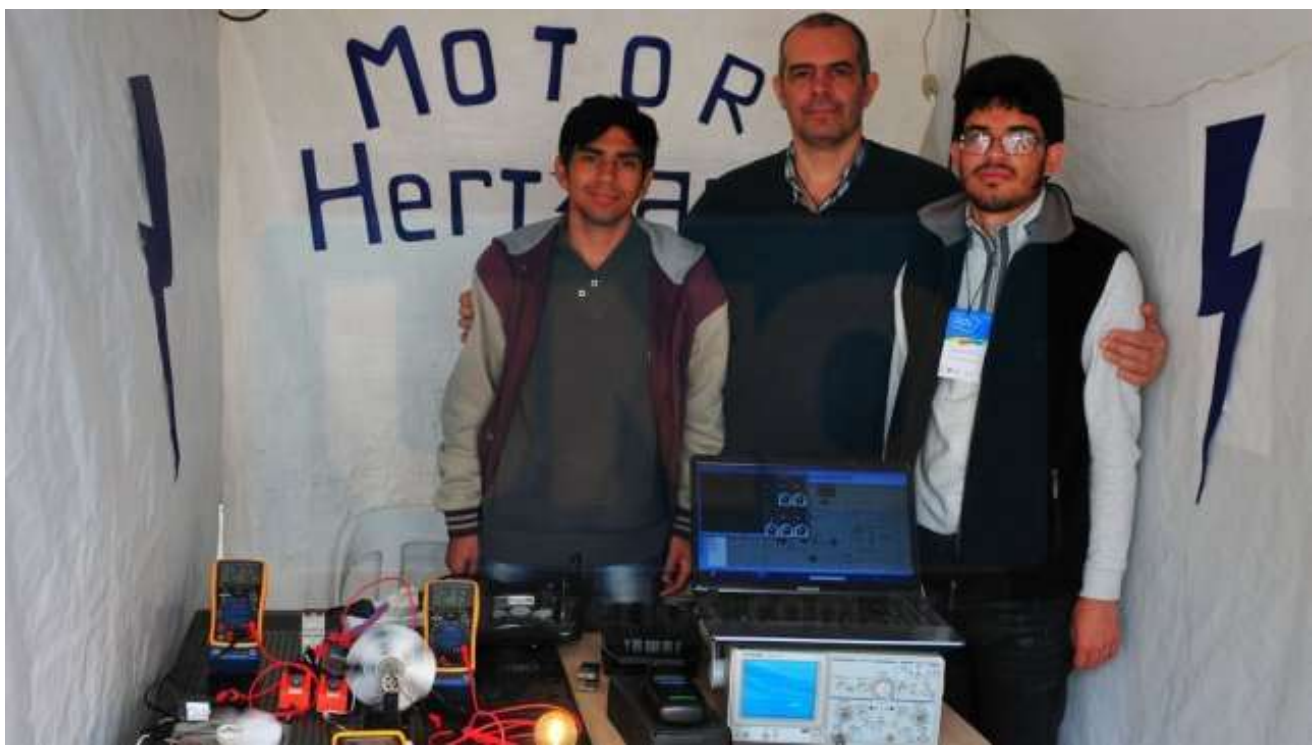

Figura 2. Reportaje periodístico diario "UNO" (Paraná, 5/10/2017), link del reportaje/entrevista: https://www. unoentrerios.com.ar/la-provincia/con-una-nutrida-propuesta-cerro-la-feria-educacion-

provincial-n1482146.htmI/Figure 2.Daily newspaper report "UNO" (Paraná, 5/10/2017), link of the report / interview:https://www.unoentrerios.com.ar/la-provincia/con-una-nutrida-propuesta-cerro -the-faireducation-provincial-n1482146.htm/

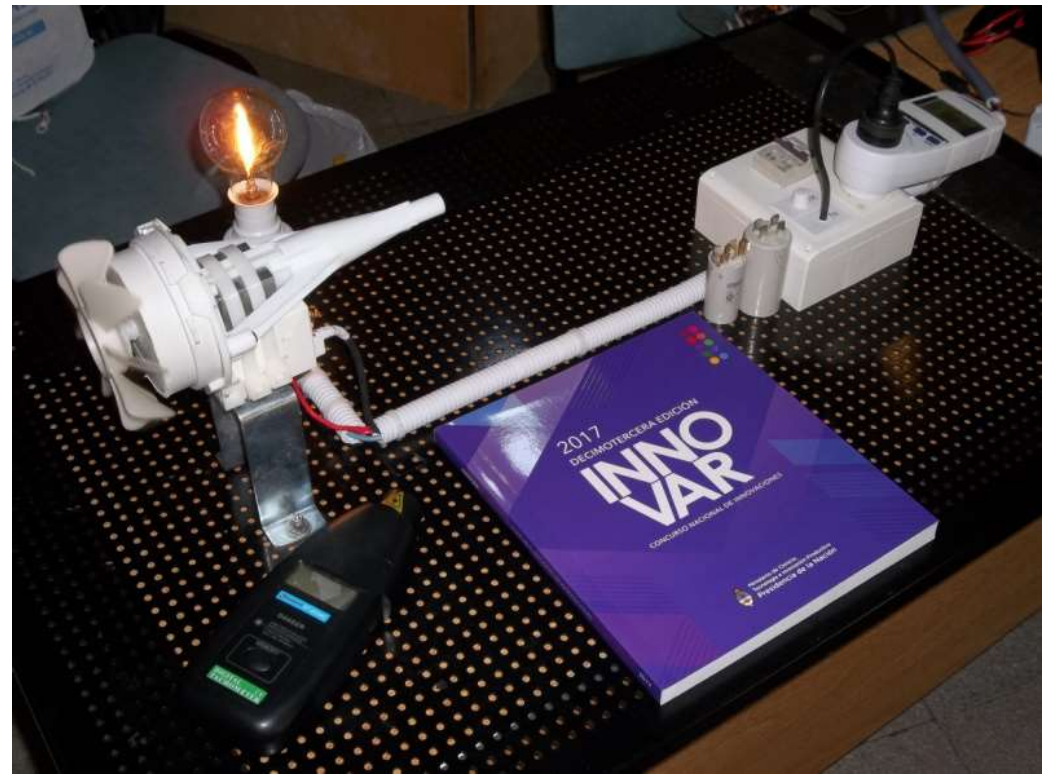

Figura 3. Catálogo Concurso nacional INNOVAR 2017 - Tecnópolis, Ministerio de Ciencia y Tecnología de la Nación. Link catálogo digital: https://mia.qob.ar/uploads/innovate/catalogo innovar 2017.pdf /Figure 3.INNOVAR 2017 National Contest Catalog - Tecnópolis, Ministry of Science and Technology of the Nation. Link digital catalog: https://mia.gob.ar/uploads/innovate/catalogo innovar 2017.pdf 
Innovación y Desarrollo Tecnológico y Social (2019) 1: 1-28

\begin{abstract}
21351 Ecomotor eléctrico sincrónico PMSM (reduce consumo energia)

Ecomotor PMSM utiliza un control de potencia de disparo por TRIAC BT137 para regulación de velocidad en los motores de inducción asincrónicos monofásicos (de tipo universal, jaula de ardilla, etc) de corriente alterna ( $A C)$, en un motor síncrono monofásico de imanes permanente (PMSM) de 220 (VAC), variando la tensión (Vrms) y la intensidad (Irms) eficaz RMS (en inglés: Root Mean Square) 0 valor cuadrático medio de la corriente eléctrica alterna; consecuentemente varía la potencia activa promedio (Pavg) monofásica (kW). Por lo que finalmente se reduce el consumo de energía eléctrica activa en un $42,8 \%$, sin que se reduzca 0 afecte la velocidad de giro (RPM) del rotor debido a la sincronía de la corriente alterna: 50 (Hertz). Esto tendría enormes ventajas en el uso de ventiladores, aires acondicionados, sistemas de refrigerador, tanto de uso doméstico como industrial.
\end{abstract}

\author{
Entre Ríos \\ 盘 Escuela Técnica $N^{0} 2$ - Independencia \\ 2. Anderson lbar Federico \\ « ibar.federico.anderson@gmail.com
}

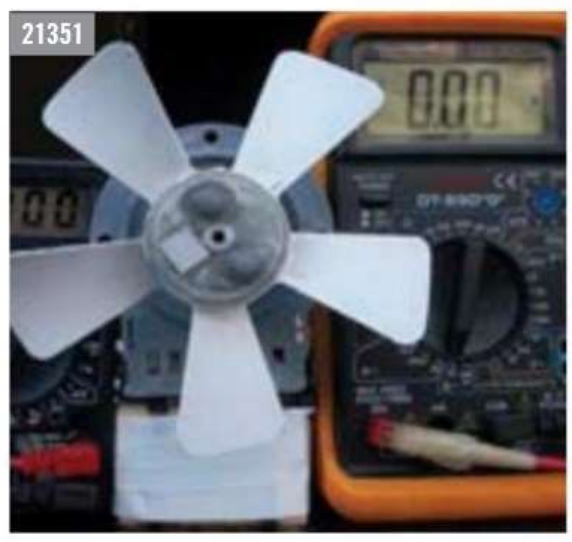

Figura 4. Ampliación de la captura de pantalla del catálogo digital INNOVAR 2017. Proyecto ID: 21351 Ecommotor eléctrico PMSM (reduce el consumo de energía). Página 206-207 del catálogo digital del Concurso Nacional INNOVAR - 2017, del Ministerio de Ciencia y Tecnología de la Nación (año 2017), ver link: https://issuu.com/innovar/docs/cat innovar2017 opt / Figure 4.Expansion of the screenshot of the INNOVAR 2017 digital catalog. Project ID: 21351 PMSM electric ecommotor (reduces energy consumption). Page 206-207 of the digital catalog of the INNOVAR National Contest - 2017, of the Ministry of Science and Technology of the Nation (year 2017), see link: https://issuu.com/innovar/docs/cat innovar2017 opt

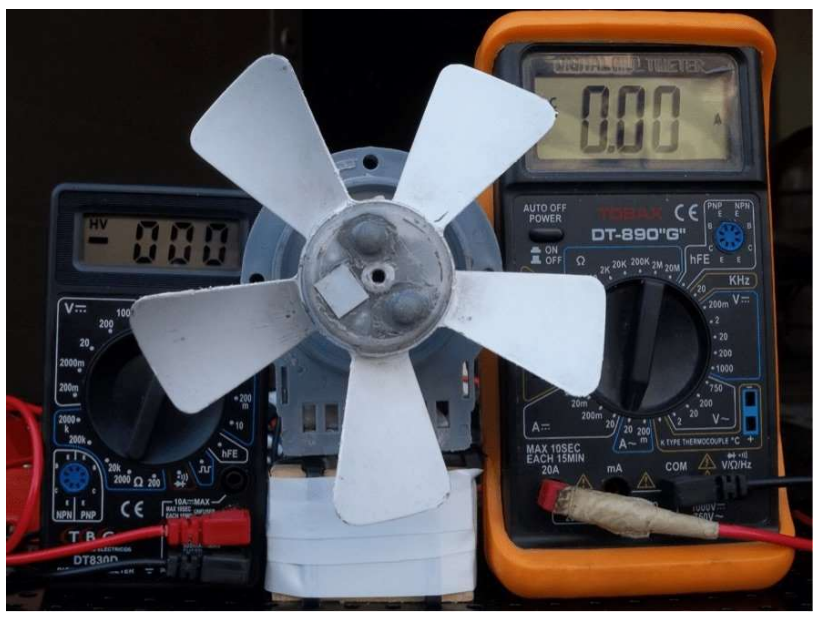

Figura 5. Portada de la presentación del proyecto "Motor Hertziano" al sitio web ECOINVENTOS. El mayor portal de habla hispana de proyectos en energías alternativas, EE y temas varios de ecología. Para ingresar a la difusión web, ver el siguiente link: https://ecoinventos.com/motor-hertziano/ / Figure 5.Cover of the presentation of the "Hertzian Motor" project to the ECOINVENTOS website. The largest Spanish-speaking portal for projects in alternative energies, EE and various topics in ecology. To access the webcast, see the following link: https://ecoinventos.com/motor-hertziano/ 


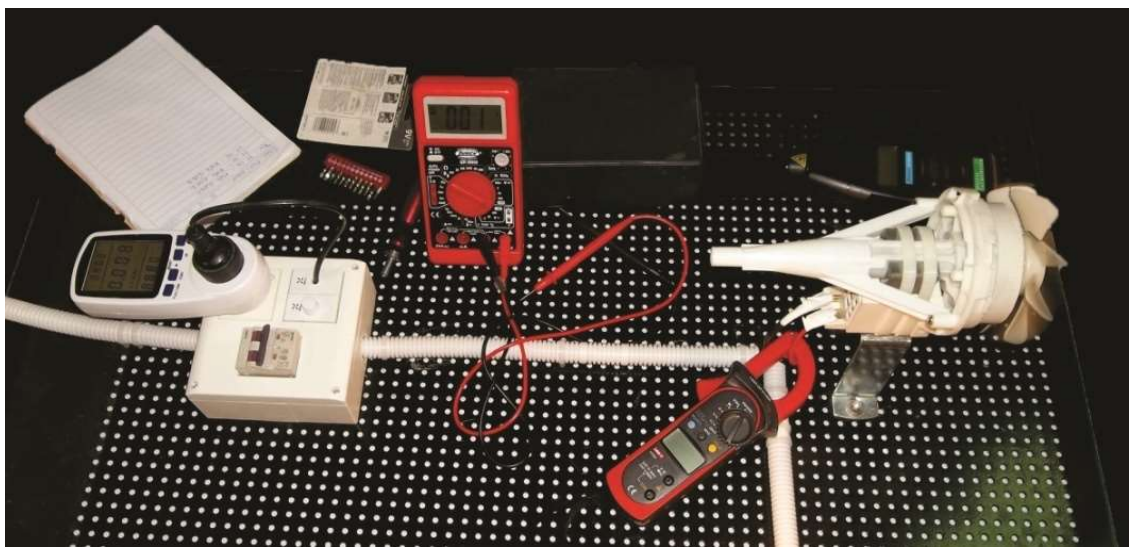

Figura 6. Presentacióndel proyecto seleccionado por el Concurso Nacional INNOVAR 2017 - Tecnópolis (Ministerio de Ciencia y Tecnología de la Nación) en el Hall de la UNER, Facultad de Ciencias de la Alimentación, Concordia. Concordia, 5/9/2018. Con motivo de la semana de la Ciencia y Tecnología de la Provincia de Entre Ríos. Se puede escuchar el audio del reportaje/entrevista realizado por el sistema integrado de radios de la UNER, Facultad de Ciencias de la Alimentación (año 2018), en el siguiente link: https://siruner.uner.edu.ar/audio/139/ecoturbina / Figure 6. Presentation of the project selected by the INNOVAR 2017 National Competition - Tecnópolis (Ministry of Science and Technology of the Nation) in the Hall of the UNER, Faculty of Food Sciences, Concordia. Concordia, 9/5/2018. On the occasion of the week of Science and Technology of the Province of Entre Ríos. You can listen to the audio report / interview conducted by the integrated radio system of UNER, Faculty of Food Sciences (year 2018), in the following link: https://siruner.uner.edu.ar/audio/139/ecoturbina

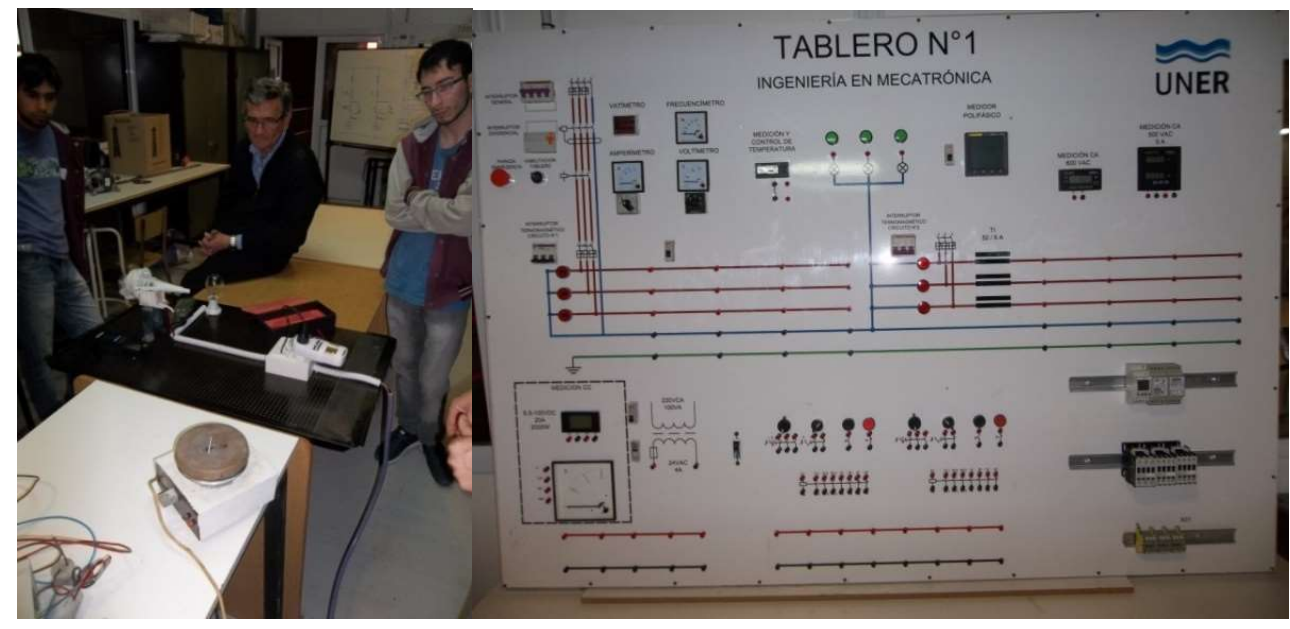

Figura 7. Adicionalmente el Proyecto fue testeado en el laboratorio de la carrera de ingeniería mecatrónica, dependiente de la UNER (Universidad Nacional de Entre Ríos). En la foto se observa al Director de la carrera: Ing. José Paramo. Link oficial a la web de la carrera: http://www.fcal.uner.edu.ar/?page id=12212/ Figure 7. Additionally, the Project was tested in the laboratory of the mechatronics engineering career, under the UNER (National University of Entre Ríos). In the photo you can see the Director of the race: Eng. José Paramo. Official link to the career website:http://www.fcal.uner.edu.ar/?page id=12212

\section{Desarrollo del producto}




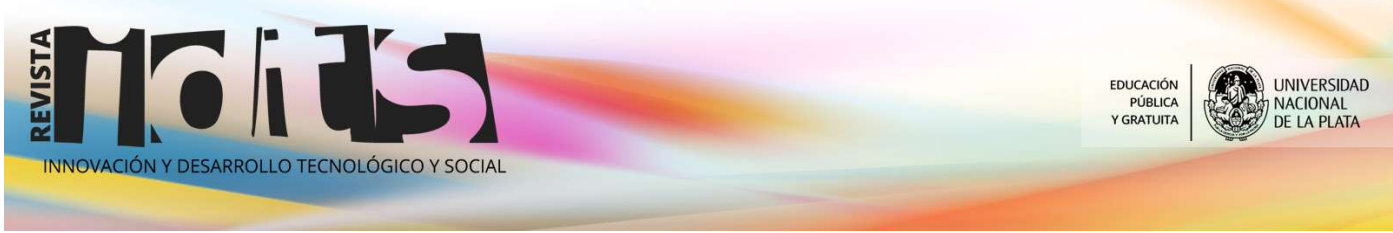

Innovación y Desarrollo Tecnológico y Social (2019) 1: 1-28

Siguiendo una metodología de Diseño Industrial sustentable (EcoDesign) combinado con Mecatrónica, las etapas bien diferenciadas fueron cinco:

1) La idea generadora (concepto proveniente de las Ciencia Física: electricidad y magnetismo).

2) Discusión en torno a la utilización de tiristores como fundamento electrónico: Triacs/SRC. Elección de la propuesta conceptual de Diseño Industrial.

3) El dibujo y/o diseño del circuito mecatrónico (eléctrico y mecánico) regulado por un control de lazo abierto electrónico.

4) La simulación electrónica computarizada, por dos (2) softwares distintos: Proteus/ISISDesign Suite 8y NI/Multisim 14.0. Se discuten también los problemas electromecánicos referidos a los motores, encontrados en la simulación CAD.

5) Fabricación de Diseño Industrial del primer prototipo (año 2017) y experimentación (primeras pruebas, ensayos de errores y verificación de datos eléctricos, electromecánicos y electrónicos).

6) Perfeccionamiento: Fabricación del segundo prototipo, mejorado (año 2018).

No debatiremos los aspectos teóricos referidos a física y electromagnetismo que sustentan el proyecto y que son de público conocimiento en los libros y en las materias de grado de cualquier carrera de especialidad técnica y/o universitaria de ingeniería y afines. Obviamente el proyecto persigue el objetivo de obtener una ventaja tecnológica diferenciada sobre productos industriales similares (ventiladores), sin violar ninguna ley de las Ciencias Físicas.

Rápidamente podemos decir que el problema se basa en una discusión sobre la utilización de ciertos componentes de materiales semiconductores para variar el voltaje eficaz (Vrms) y la intensidad de la corriente eficaz (Irms) suministrada al motor. Dado que esta es una técnica que solo funciona para motores asincrónicos, nosotros la utilizaremos en un motor sincrónico (innovación).

Para ello, se necesita muy poca electrónica. Si bien el torque decae, se trabaja hasta un límite de no exceder el par-motor o momento de torsión crítico del motor (en el cual el rotor se detendría y comenzaría a vibrar recalentando al estator). El punto crítico del momento de torsión mínimo-máximo se ajusta por un potenciómetro.

El control de media onda para la corriente eléctrica alterna con un (1) SCR (SiliconControlledRectifier, traducido como: rectificador controlado de silicio) hacia la carga y el control de onda completa para la corriente eléctrica alterna con dos (2) SCRs o un (1) Triac; según el Ing. Gilberto Enríquez Harper, en su libro El ABC de las máquinas 


\section{INNOV}

Innovación y Desarrollo Tecnológico y Social (2019) 1: 1-28

eléctricas. III Instalación y control de motores de corriente alterna, capítulo 5 "Fundamentos de electrónica de potencia", se obtiene del siguiente modo:
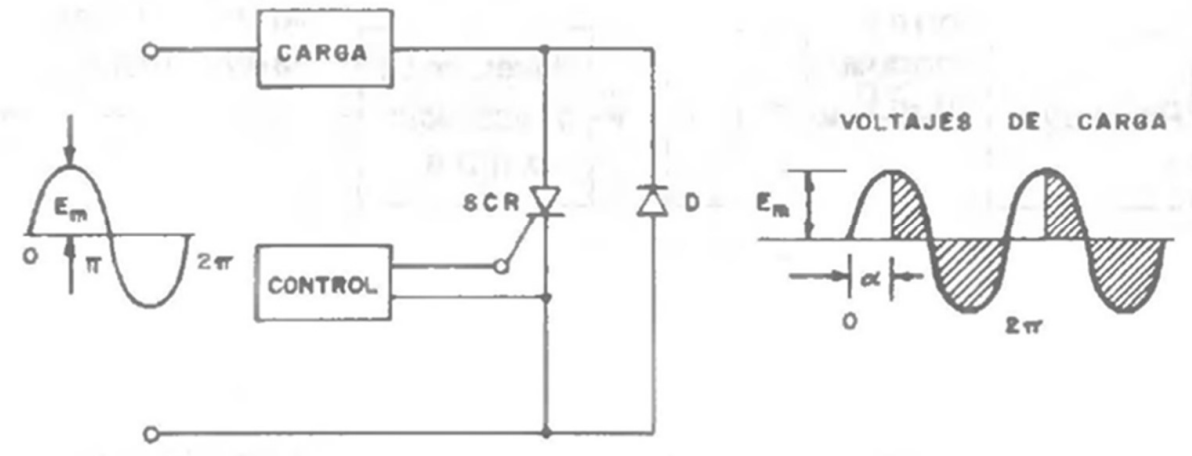

Figura 8. Control de media onda con un (1) solo dispositivo SCR. Fuente: Harper EI ABC de las máquinas eléctricas. III Instalación y control de motores de corriente alterna, capítulo 5 "Fundamentos de electrónica de potencia". / Figure 8. Half wave control with one (1) single SCR device. Source: Harper The ABC of electric machines. III Installation and control of alternating current motors, chapter 5 "Fundamentals of power electronics".

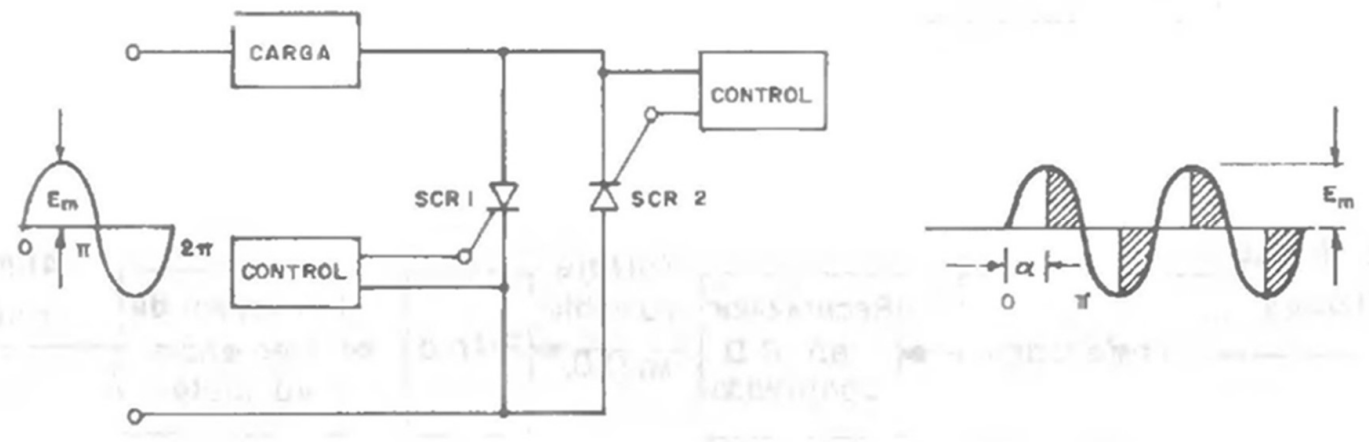

Figura 9. Control de onda completa con dos (2) SCRs.Fuente: Harper El ABC de las máquinas eléctricas. III Instalación y control de motores de corriente alterna, capítulo 5 "Fundamentos de electrónica de potencia". / Figure 9. Full wave control with two (2) SCRs. Source: Harper The ABC of electric machines. III Installation and control of alternating current motors, chapter 5 "Fundamentals of power electronics".
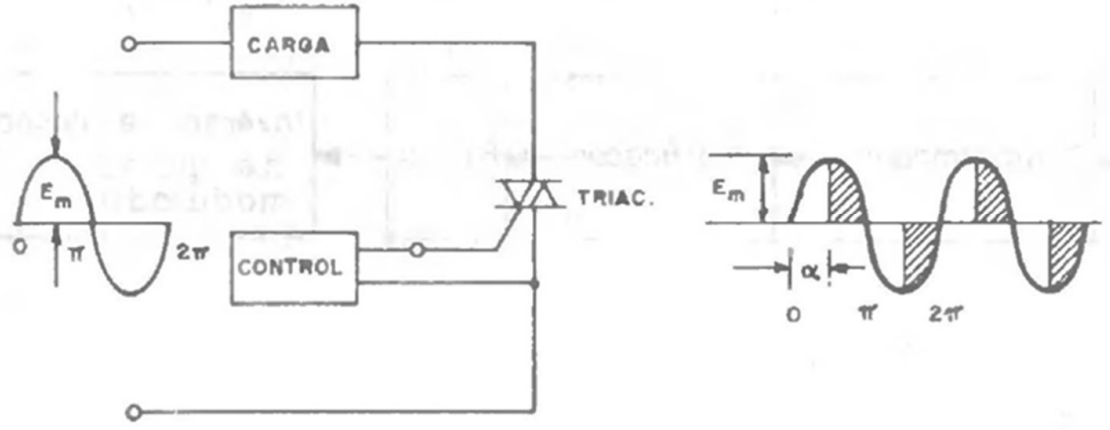


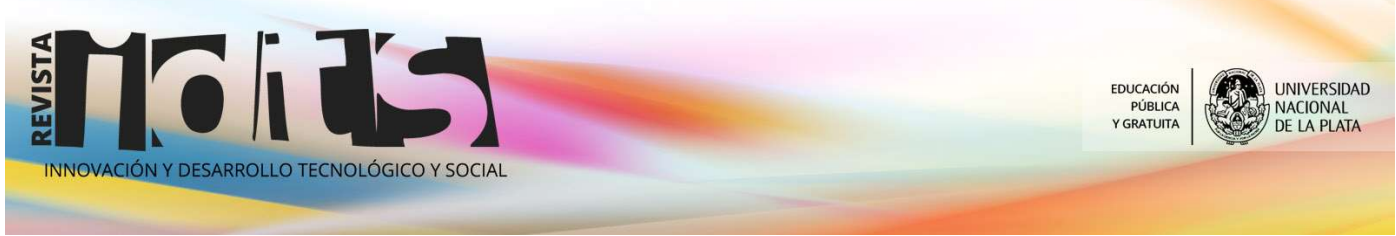

Innovación y Desarrollo Tecnológico y Social (2019) 1: 1-28

Figure 10.Control de onda completa con un (1) Triac, equivalente a dos (2) SCRs conectados en atiparalelo. Fuente: HarperEl ABC de las máquinas eléctricas. III Instalación y control de motores de corriente alterna, capítulo 5 "Fundamentos de electrónica de potencia". / Figure 10. Full wave control with one (1) Triac, equivalent to two (2) SCRs connected in ati-parallel. Source: Harper The ABC of electric machines. III Installation and control of alternating current motors, chapter 5 "Fundamentals of power electronics".

Un $\operatorname{Triac}^{29}$ o Triodo para Corriente Alterna (AC) es un dispositivo semiconductor, de la familia de los tiristores. La diferencia con un tiristor convencional es que éste es unidireccional y el Triac es bidireccional. De forma coloquial podría decirse que el Triac es un interruptor capaz de conmutar la corriente alterna. Su estructura interna se asemeja en cierto modo a la disposición que formarían dos SCR (en inglés SCR: Silicon Controlled Rectifier) o Rectificador Controlado de Silicio, conectado en direcciones opuestas.

Ante la evidente ventaja de un Triac frente a dos SCR, aquí se ha seleccionado un circuito de control disparador con Triac mucho más simple. El Triac controla el paso de la corriente alterna a la carga conmutando entre los estados de conducción (pasa corriente) y corte (no pasa corriente) durante los semiciclos negativos y positivos de la señal de alimentación (220 VAC) de la señal de corriente alterna. El Triac se dispara cuando el voltaje entre el capacitor y el potenciómetro (conectado a la compuerta del Triac) sea el adecuado.

Puede asimilarse la conformación de un Triac como dos SCR conectados en antiparalelo con un sólo terminal externo de control, compuerta, mediante el cual se produce el encendido en cualquier dirección. Es decir, con cualquier sentido de circulación de corriente en compuerta se logra la conducción del dispositivo. También al igual que para un SCR, la presencia de corriente de compuerta hace que el voltaje de ruptura disminuya tanto más, cuanto más aumente la corriente aplicada.

Los Diac son una denominación de tiristor, y se usan normalmente para autocompletar el ritmo variado del disparo de un TRIAC, otra clase de tiristor. Si tomamos un diseño de circuito de disparo por Triac, el Diac presente en el diseño del circuito, solo es un elemento constitutivo del circuito de disparo para obtener la transición del estado de bloqueo al de conducción. El Diac (Diodo para Corriente Alterna) es un dispositivo semiconductor doble de dos conexiones, es un diodo bidireccional autodisparable que conduce la corriente sólo tras haberse superado su tensión de disparo alternativa, y mientras la corriente circulante no sea inferior al valor triple de voltios característico para ese dispositivo. La mayoría de los Diac tienen una tensión de disparo doble variable de alrededor de 30 (Voltios).

\footnotetext{
29 "Triac", Wikipedia, 2019 [En línea]. Disponible https://es.wikipedia.org/wiki/Triac\#/media/File:Circuito Dimmer (atenuador de luz) para una red de 2 20V - $50 \mathrm{~Hz} . j p g$ [Accedido: 25-ene-2019]
} 
El Triac se usa para controlar el flujo de corriente promedio a una carga, con la particularidad de que conduce en ambos sentidos y puede ser bloqueado por inversión de la tensión o al disminuir la corriente por debajo del valor de mantenimiento. El Triac puede ser disparado independientemente de la polarización de puerta, es decir, mediante una corriente de puerta positiva o negativa.

Hay que aclarar que el capacitor en un circuito de corriente alterna (como éste) tiene su voltaje atrasado con respecto a la señal original. Cambiando el valor del potenciómetro, se modifica la razón de carga del capacitor, el atraso que tiene y por ende el desfase con la señal alterna original. Esto permite que se pueda tener control sobre la cantidad de corriente que pasa a la carga y así la potencia que en ésta, se va a consumir.

Una de las aplicaciones que tiene en el control de motores de corriente alterna, se encuentra en el control de velocidad de los motores universales, permitiendo un amplio rango de control de velocidad así como un comportamiento suave del motor. Estas características deseables se obtienen con el uso del Triac para el control de onda completa con una red de desfasamiento de doble fase.

A continuación mostramos un diseño de circuito de control del autor Ing. Gilberto Enriquez Harper:

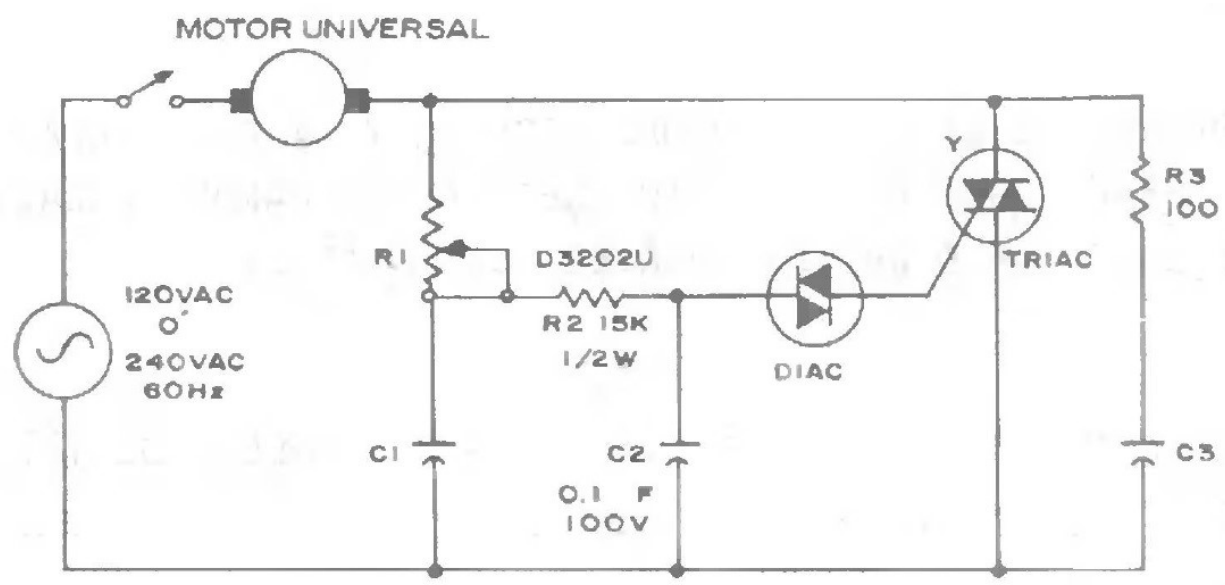

Figure 11. Control de onda completa con un (1) Triac, equivalente a dos (2) SCRs conectados en antiparalelo. Los componentes para 240 (VCA) a $60(\mathrm{~Hz})$ son: $R 1=200(\mathrm{k}) / 1(\mathrm{~W}), C 1=0.1(\mu \mathrm{F}) / 400(\mathrm{~V})$, C3=0.22 ( $\mu \mathrm{F}) / 400(\mathrm{~V})$ y Triac T2700D. Fuente: Harper El ABC de las máquinas eléctricas. III Instalación y control de motores de corriente alterna, capítulo 5 "Fundamentos de electrónica de potencia". / Figure 11. Full wave control with one (1) Triac, equivalent to two (2) SCRs connected in anti-parallel. The components for $240(\mathrm{VCA})$ to $60(\mathrm{~Hz})$ are: $R 1=200(\mathrm{k}) / 1(\mathrm{~W}), \mathrm{C1}=0.1(\mu \mathrm{F}) / 400(\mathrm{~V}), \mathrm{C3}=0.22(\mu \mathrm{F}) / 400(\mathrm{~V})$ and Triac T2700D. Source: Harper The ABC of electric machines. III Installation and control of alternating current motors, chapter 5 "Fundamentals of power electronics". 


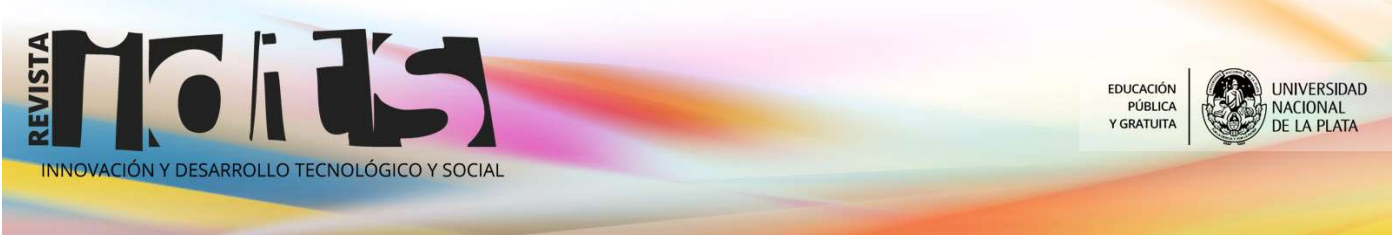

Innovación y Desarrollo Tecnológico y Social (2019) 1: 1-28

El Triac proporciona rectificación de onda completa (como dos SCRs conectados "espalda a espalda" pero sin las complicaciones de las compuertas de los SCRs). El voltaje que se entrega a la carga es siempre senoidal.

La gráfica del recorte de la onda, producto del disparo del Triac, asociado a la velocidad obtenida por el motor universal de AC.
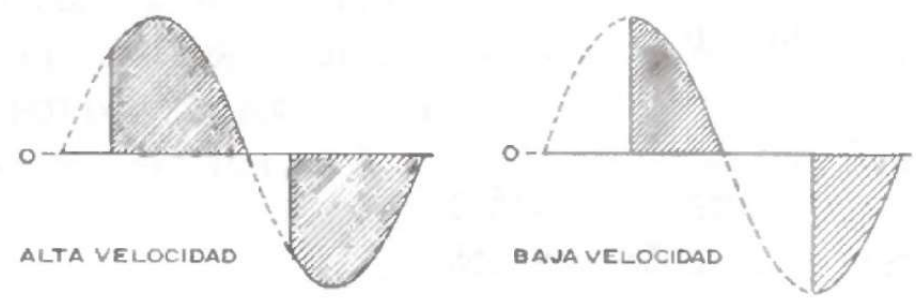

Figure 12. En el gráfico se muestra la asociación del troceado de la onda, producto del ángulo de disparo del Triac, con la velocidad del motor universal a-sincrónico. Fuente: Harper El ABC de las máquinas eléctricas. III Instalación y control de motores de corriente alterna, capítulo 5 "Fundamentos de electrónica de potencia". / Figure 12. The graph shows the association of the splitting of the wave, product of the Triac trigger angle, with the speed of the universal a-synchronous motor. Source: Harper The ABC of electric machines. III Installation and control of alternating current motors, chapter 5 "Fundamentals of power electronics".

Esta técnica de control resulta apropiada cuando la carga es un ventilador (o un extractor de aire), en donde un pequeño cambio en la velocidad produce un cambio relativamente grande en la velocidad del aire. Otro aspecto favorable de estas cargas es su requerimiento de bajo par de arranque.

Terminado el debate en cuanto al diseño más conveniente., se pasó a desarrollar el primer prototipo (año 2017) en simultáneo con las simulaciones electromecánicas y electrónicas; realizando ajustes experimentales, tareas diversas de correcciones sobre la marcha del experimento, prototipos parciales de partes y diversos modelos de prototipos (variantes). A todo esto se le sumó las visitas a los bancos de prueba de los laboratorios de Ingeniería (eléctrica y mecatrónica) de diversas Universidades Nacionales: UNER (Universidad Nacional de Entre Ríos, sede Concordia, Provincia de Entre Ríos) y UTN (Universidad Tecnológica Nacional, sede Concordia, Provincia de Entre Ríos), también al LIDDI (Laboratorio del Departamento de Diseño Industrial de la Universidad Nacional de La Plata, sede La Plata, Provincia de Buenos Aires). Debates con profesionales diversos: ingenieros eléctricos y electrónicos, diseñadores industriales, profesores universitarios de grado y posgrado, colegas, etcétera.

La actividad principal llevada a cabo para la construcción del primer prototipo fue: adoptar un motor sincrónico de tipo PMSM de 30 watts de potencia nominal (con rotor 


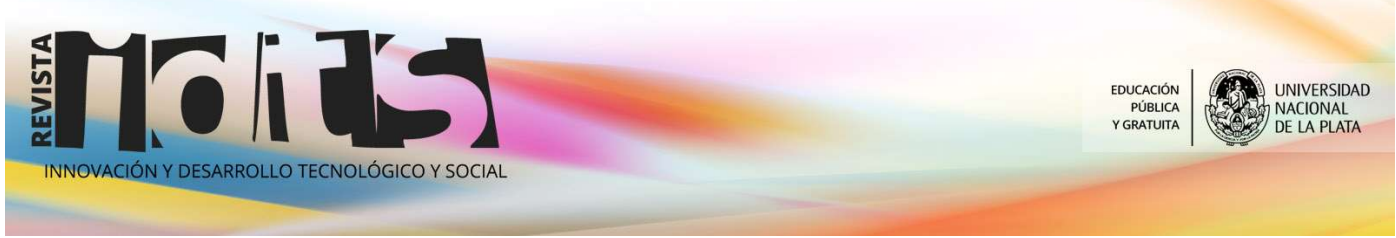

Innovación y Desarrollo Tecnológico y Social (2019) 1: 1-28

de imanes permanentes de ferrite) obtenido a partir del estator de una electrobomba de lavarropas y acoplarlo a las paletas de un rotor de un motor a-sincrónico de espiras de sombra (o espira de fragger) de microonda. Lo que definió la electromecánica del motor.

Específicamente, para el desarrollo del segundo prototipo se debió adaptar un motor sincrónico de tipo PMSM (con rotor de imanes permanentes de ferrite), obtenido a partir del estator de una electrobomba de lavavajillas de 65 (watts) de potencia nominal y acoplarlo a las paletas del mismo rotor de un motor a-sincrónico de espiras de sombra (o espira de fragger).

Controlándolos a ambos prototipos con la misma electrónica de potencia de disparo por Triac BT137. Tal cual la bibliografía típica -que es abundante- de control de motores de corriente eléctrica alterna $(A C)$ lo describe y que ya hemos citado a modo referencial ${ }^{30}$.

Como ya se aclaró, en este trabajo se utilizó un autor específico en control de motores de corriente alterna (Harper, 2006). En efecto, el autor en su capítulo de control electrónico de corriente alterna, del libro El $A B C$ de las máquinas eléctricas III. Instalación y control de motores de corriente alterna[20]. Detalla el circuito típico utilizado en estos casos [21].

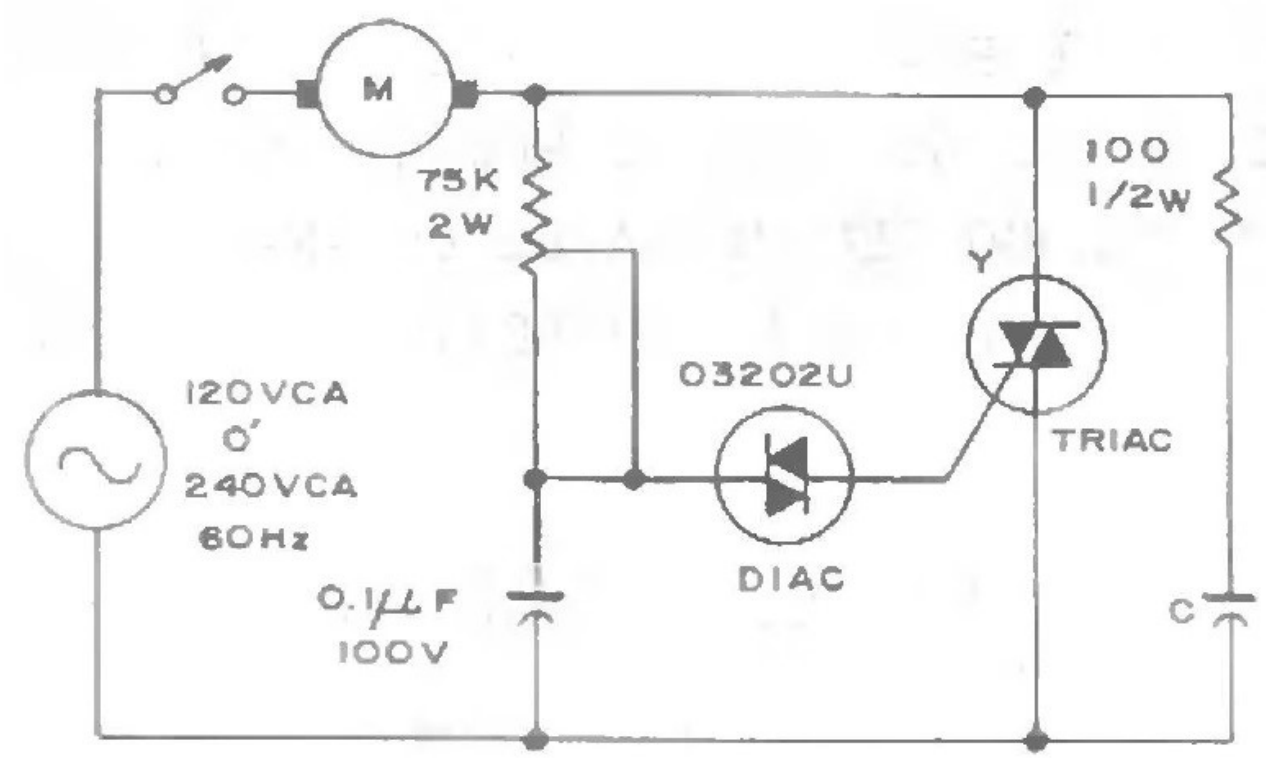

Figura 13. Dibujo de circuito según el capítulo 6 de control electrónico de potencia del motor de inducción, del libro de Harper: El ABC de las máquinas eléctricas III. Instalación y control de motores de corriente alterna. El motor de inducción a-sincrónico (o a-síncrono), simbolizado por la letra (M), se

${ }^{30} \mathrm{Harper}, \mathrm{G}$. "Capítulo 6: Control electrónico de motores eléctricos". En El ABC de las máquinas eléctricas III. Instalación y control de motores de corriente alterna (pp.355-370). México: Grupo Noriega Editores, 2006. 
debe reemplazar por un motor sincrónico (o síncrono) de tipo PMSM (PermanentMagnetSynchronous Motor, o motor síncrono de imán permanente). /Figure 13. Circuit drawing according to chapter 6 of electronic control of power of the induction motor, from Harper's book: The ABC of electrical machines III. Installation and control of AC motors. The a-synchronous (or a-synchronous) induction motor, symbolized by the letter (M), must be replaced by a synchronous (or synchronous) motor of the PMSM type (Permanent Magnet Synchronous Motor, or permanent magnet synchronous motor).

En simultáneo con la construcción del primer el prototipo se realizó la simulación eléctrica en Proteus Design Suite $8 C A D^{31}$, un software de automatización de diseño electrónico, desarrollado por Labcenter Electronics Ltd., que consta de los dos programas principales: Ares e Isis, y el módulo VSM. El Programa ISIS, Intelligent Schematic Input System (o Sistema de Enrutado de Esquemas Inteligente) permite diseñar el plano eléctrico/electrónico del circuito que se desea realizar con componentes muy variados como se muestra a continuación. Siguiendo algunas especificaciones generales, según G. Harper, para el diseño de este tipo de circuito eléctrico / electrónico.

Existen muchas variantes típicas, análogas al diseño propuesto por Harper, para el diseño de estos circuitos eléctricos/electrónicos que controlan una carga R-L (motor) desde 300 (W) hasta 5000 (W); indistintamente se trate de un motor de inducción asincrónico R-L o una carga resistiva pura (foco de luz) [22]. Rango suficiente de potencia para su utilización en electrodomésticos domiciliarios y/o comerciales (no industriales). En este proyecto se utilizó específicamente la siguiente variante de diseño de circuito eléctrico/electrónico R-L-C:

31"Suite de Diseño Proteus", 2019. [En línea]. Disponible en: https://www.labcenter.com [Accedido: 25-ene2019] 


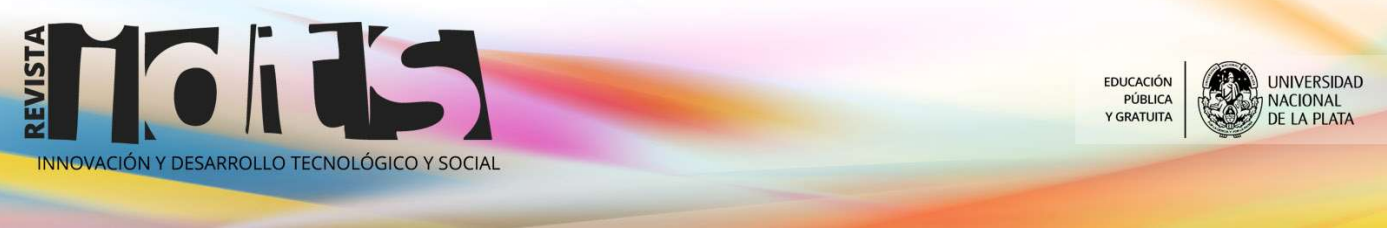

Innovación y Desarrollo Tecnológico y Social (2019) 1: 1-28

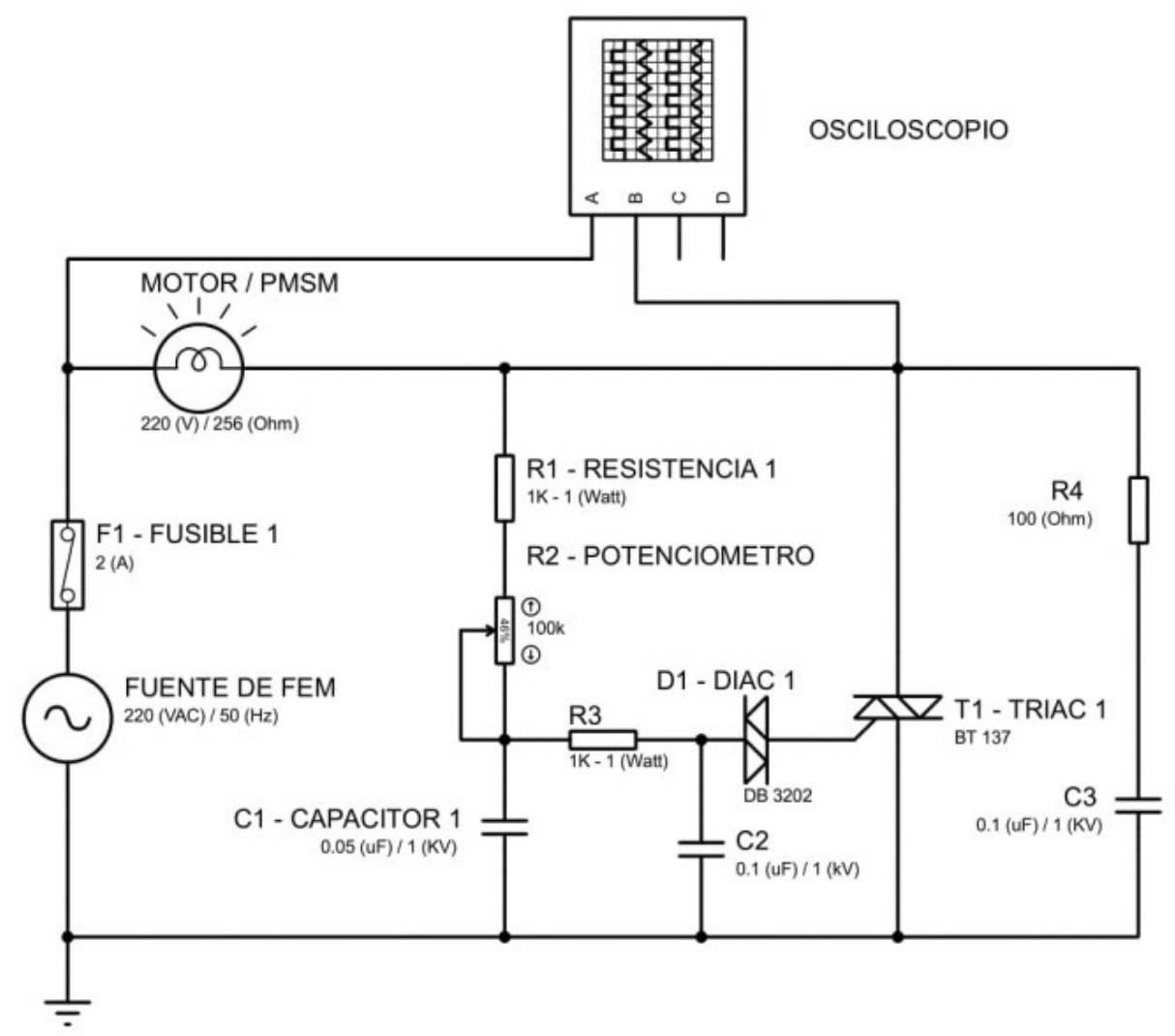

Figura 14. Diseño del circuito electrónico R-L-C de control de potencia del motor AC sincrónico de tipo PMSM (Permanent Magnet Synchronous Motor) o motor síncrono de imanes permanentes, con una de impedancia de $256(\Omega)$, simulado con el Proteus Design Suite 8 CAD. El control se efectúa por un resistor variable mecánico (potenciómetro), dado que al variar la resistencia se puede controlar el ángulo de conducción. Se observa la fuente de fem (fuerza electro-motriz) de 220 (VAC), 50 (Hz), el Diac conectado al Triac BT 137 y el osciloscopio del software Proteus Design Suite 8 CAD. Fuente: elaboración propia. / Figura 14. Design of the electronic circuit R-L-C of power control of the synchronous AC motor of PMSM type (Permanent Magnet Synchronous Motor) or synchronous motor of permanent magnets, with an impedance of $256(\Omega)$, simulated with the Proteus Design Suite $8 C A D$. The control is carried out by a mechanical variable resistor (potentiometer), since the resistance can be controlled by varying the resistance. The source of emf (electromotive force) of $220(\mathrm{VAC}), 50(\mathrm{~Hz})$, the Diac connected to the Triac BT 137 and the oscilloscope of the Proteus Design Suite 8 CAD software are observed. Source: selfmade.

Se debe aclarar que por las características del tipo de diseño del circuito que combina aspectos electrónicos con electromecánicos (motor), se tuvieron problemas con la utilización del software de simulación: Proteus Design Suite 8 CAD. Este software está bien adaptado para la simulación eléctrica y electrónica, pero no para los aspectos electromecánicos en corriente alterna (AC); pues, todos los motores disponibles en el paquete informático son para aplicaciones de corriente continua (DC). Por lo que la carga (load) se simuló con una circuito $\mathrm{R}$ (resistivo puro)equivalente a la impedancia de un circuito R-L de un motor síncrono (o sincrónico).Por otro lado, también se buscó en el 
Innovación y Desarrollo Tecnológico y Social (2019) 1: 1-28

software NI/Multisim $14.0^{32}$ motores sincrónicos de imanes permanentes (PMSM), ya que dispone de un paquete de aplicaciones muy bueno para el diseño de motores de corriente alterna (AC) y como factor adicional, sus instrumentos de medición son mejores para este tipo de aplicaciones de desarrollo, que los disponibles por el Proteus Design Suite 8 CAD.A continuación, se ilustra la simulación obtenida con el diseño del circuito de la figura 8, para ser visualizada en el osciloscopio AgilentXSC 3 [] del software NI Multisim 14.0 []; que presenta una mejor visualización de la onda completa y recortada (disparo del Triac) para el mismo tipo de diseño de circuito eléctrico/electrónico anteriormente simulado en el Proteus Design Suite 8 CAD.
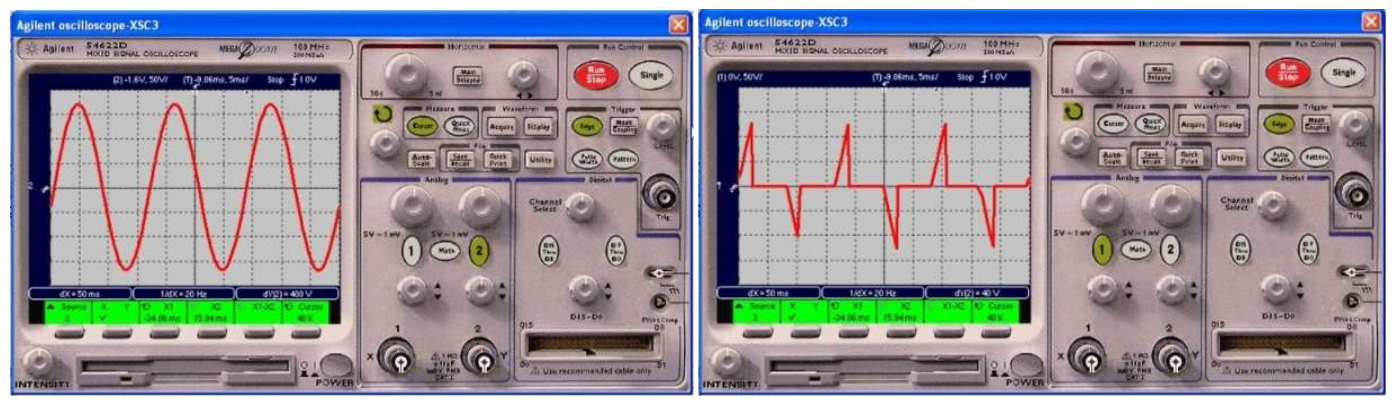

Figuras 15(A) y (B).A mayor potencia activa (imagen izquierda) el motor sincrónico utiliza el total de la onda sinusoidal de la tensión (Vrms) e intensidad de la corriente (Arms). Pero en el motor síncrono (o sincrónico), cuando la onda sinusoidal es recortada por el disparo del Triac (imagen de la derecha); desciende el consumo de energía activa ( $k W h$ ) por reducción de la potencia promedio o media activa (Pmed) medida en vatios (Watts). Simulación del circuito conectado al osciloscopio Agilent XSC $3^{33}$, del software NI Multisim 14.0. Fuente: elaboraciónpropia. / Figures 15 (A) and (B). At higher active power (left image) the motor uses the total of the sine wave voltage (Vrms) and current intensity (Arms). But in the synchronous (or synchronous) motor, when the sine wave is trimmed by the Triac shot (right image); The active energy consumption ( $k W h$ ) decreases by reduction of the average or active average power (Pmed) measured in Watts. Simulation of the circuit connected to the Agilent XSC 3 oscilloscope, from NI Multisim 14.0 software. Source: selfmade.

Aunque, en el caso del software NI Multisim 14.0, si bien en el paquete de opciones de máquinas electromecánicas de corriente alterna, existen varios tipos de motores para ser utilizados; el problema es que no se dispone de una máquina sincrónica monofásica de imanes permanentes del tipo PMSM (Synchronous Machine Permanent Magnets), necesitada para la simulación. La única máquina sincrónica disponibles de imanes permanentes es trifásica (no monofásica) y como tal no resultóser de utilidad para este trabajo. Esta falta del know-how específico para afrontar este inconveniente, conforma

32"¿Qué es Multisim ${ }^{\mathrm{TM}}$ ? Multisim ${ }^{\mathrm{TM}}$ es un software estándar en industria para diseño de circuitos y simulación SPICE para electrónica de potencia, analógica y digital en la educación y la investigación.", 2019. [En línea]. Disponible en: http://www.ni.com/es-cr/shop/electronic-test-instrumentation/application-software-forelectronic-test-and-instrumentation-category/what-is-multisim.html [Accedido: 25-ene-2019]

33MahtabZ, "Instrumentos Multisim", 07-jul-2011. [Blog d la comunidad de diseño de circuitos de National Instruments] https://forums.ni.com/t5/National-Instruments-Circuit/Multisim-Instruments/ba-p/3489007 [Accedido: 25-ene-2019] 


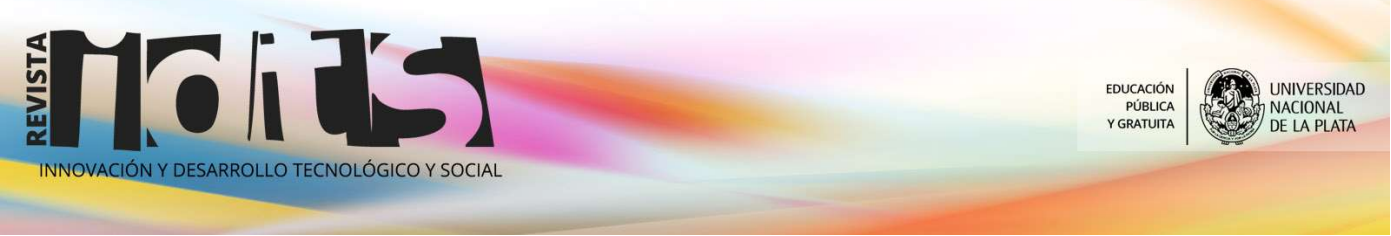

Innovación y Desarrollo Tecnológico y Social (2019) 1: 1-28

un problema propio de la etapa de Investigación + Desarrollo tecnológico (I+D) que se debió afrontar y superar con el diseño de un circuito equivalentes R-C-L.

Se debe recordar que todo motor eléctrico a-sincrónico, en máxima velocidad angular utiliza mayor porción de la onda sinusoidal de la tensión (voltios) e intensidad de la corriente (amperios) y cuando trabaja con menor tensión e intensidad de la corriente utiliza una menor porción de la onda sinusoidal. Pero en el motor síncrono (o sincrónico), cuando la onda sinusoidal es recortada por el disparo del Triacy desciende el consumo de energía activa ( $\mathrm{kWh}$ ) por reducción de la potencia media activa (Watts) debido al troceado de la onda sinusoidal (disparo del Triac), el motor no pierde velocidad como sucede con los motores a-sincrónicos.

Tabla 1. Los valores de tensión (Vrms) e intensidad (Irms) de la corriente eléctrica alterna (AC) y coseno de fi $(\cos \phi)$, oscilando a $50(\mathrm{~Hz})$ que se indican en la siguiente tabla; se tomaron en los contactos de ingreso al circuito $R$ - $L$ del devanado inductor (estator) en la máquina rotativa (motor sincrónico). Los valores de Potencia media activa (Watts) y consumo de energía activa ( $k W h$ ) se tomaron en la entrada de f.e.m. (fuerza electro motriz) en todo el circuito R-L-C (electromecánico y electrónico). No se ha podido obtener y/o especificar la información referida a la potencia reactiva. Fuente: elaboraciónpropia. / Table 1. The values of voltage (Vrms) and intensity (Irms) of the alternating electric current (AC) and cosine of fi (cos.), oscillating at $50(\mathrm{~Hz})$ that are indicated in the following table; were taken in the input contacts to the $R$ - $L$ circuit of the inductor winding (stator) in the rotating machine (synchronous motor). The values of active average power (Watts) and active energy consumption ( $k W h$ ) were taken at the entrance of f.e.m. (electro motive force) throughout the R-L-C circuit (electromechanical and electronic).It has not been possible to obtain and / or specify the information related to the reactive power.GSource: selfmade.

\begin{tabular}{cccccc}
\hline $\begin{array}{c}\text { Control de potencia } \\
\text { (Triac) }\end{array}$ & $\begin{array}{c}\text { Consumo } \\
\text { energía } \\
\text { activa } \\
\text { (kWh) }\end{array}$ & $\begin{array}{c}\text { Potencia } \\
\text { media activa } \\
\text { (Watts) }\end{array}$ & $\begin{array}{c}\text { Intensidad } \\
\text { corriente } \\
\text { (Irms) }\end{array}$ & $\begin{array}{c}\text { Tensión } \\
\text { (Vrms) }\end{array}$ & $\begin{array}{c}\text { Coseno fi } \\
\text { (cos } \phi)\end{array}$ \\
\hline $\begin{array}{c}\text { Sin ecorte de onda } \\
\text { (Triac apagado) }\end{array}$ & 0.025 & 25.1 & 0.88 & 220 & 0.13 \\
$\begin{array}{c}\text { Recorte de onda máximo } \\
\text { (Triac en disparo } \\
\text { máximo) }\end{array}$ & 0.012 & 13 & 0.21 & 77 & 0.94 \\
\hline
\end{tabular}

La fórmula para la potencia media activa (Pmed), en un circuito R-C-L general de corriente alterna (CA) es igual al producto de la tensión eficaz (Vrms), por la intensidad eficaz de la corriente eléctrica (Irms), multiplicado por el factor de potencia o coseno de fi: $\cos (\phi)$. Exactamente, según algunos autores ${ }^{34}$ clásicos de física, electricidad y magnetismo: $\quad$ "Pmed $=\frac{1}{2}$ VI $\cos (\phi)=V r m s \cdot \operatorname{Irms} \cdot \cos (\phi) " \quad$ (Sears-Zemansky,

\footnotetext{
${ }^{34}$ Sears; Zemansky. "31: Corriente alterna", Física Universitaria con Física Moderna, Volumen 2, México: Pearson Educación, 2009, pp. 1061-1091 [En línea]. Disponible en: https://www.ucursos.cl/usuario/42103e5ee2ce7442a3921d69b0200c93/mi blog/r/Fisica General -

Fisica Universitaria Vol 2 ed 12\%28Sears-Zemansky\%29.pdf [Accedido: 25-ene-2019]
} 


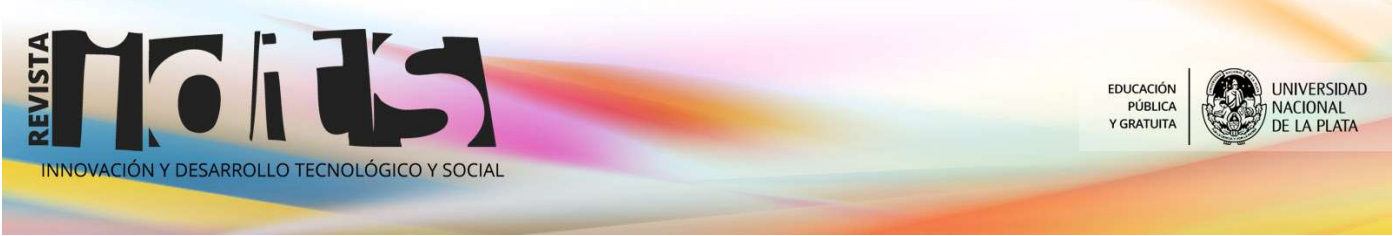

Innovación y Desarrollo Tecnológico y Social (2019) 1: 1-28

2009:1076). Valores que se tomaron con los instrumentos correspondientes de verdadero valor eficaz o RMS (Root Means Square).

Dependiendo donde se localicen los instrumentos de medición en el diseño del circuito y se tomen los datos; los valores de tensión (voltaje), intensidad de la corriente (amperaje), potencia media activa (vatios) y coseno de fi, son todos datos que fluctúan. En la siguiente tabla comparativa se observan esas variaciones -fluctuaciones- en función del estado de operabilidad del Triac (apagado y encendido).

Luego, considerando la estabilidad de la frecuencia (Herzios) de la corriente alterna (AC) en la República Argentina que es de 50 (Hertz); lo que asegura un giro constante a 3000 RPM (revoluciones por minutos) del eje del motor. Si el par de juego de polos de la máquina síncrona es equivalente a dos (2) polos (norte-sur) en el estator. Siendo $p=2$, la cantidad de polos utilizados en el diseño del prototipo -según autores ${ }^{35}$ en la materia máquinas eléctricas- posee la fórmula siguiente:

El rotor y el estator siempre tienen el mismo número de polos (...), el número de polos determina la velocidad síncrona del motor:

$n s=120 * f / p$

Donde:

$n s=$ velocidad del motor $(r / m i n)$

$f=$ frecuencia de la fuente $(\mathrm{Hz})$

$p=$ número de polos (Wildi, 2019:379)

Cálculo con el que se obtienen el dato constante de las revoluciones por minuto ( $\mathrm{r} / \mathrm{min}$ o RPM), según la frecuencia de la corriente en la República Argentina: ns $=120 * 50$ $(\mathrm{Hz}) / 2$ = 3000 (r/min), o 3000 (RPM).

Las 3000 (revoluciones/minutos) o 3000 (RPM), como ya se indicó con anterioridad, es consecuencia de la frecuencia de la corriente alterna (AC). En efecto, el prototipo de la Eco-turbina no disminuye sus RPM cuando se reduce el consumo de energía activa de la turbina (ergo: desciende su potencia activa). Esto se logra manteniendo no-constante el control escalar voltios/hertz $(\mathrm{V} / \mathrm{Hz})$, típico de los variadores de frecuencia (VDF) o drivers.

No sucede lo mismo con el torque, dado que este desciende hasta el límite mínimo, sin que afecte la capacidad de las aspas del rotor de realizar trabajo mecánico sobre el aire.

${ }^{35}$ Wildi, T. "17: motores síncronos", en Máquinas eléctricas y sistemas de potencia, México: Pearson Educación, 2007, p. 377-398 [En línea]. Disponible en: https://www.academia.edu/31911167/Maquinas Electricas y Sistemas de Potencia [Accedido: 25-ene2019] 


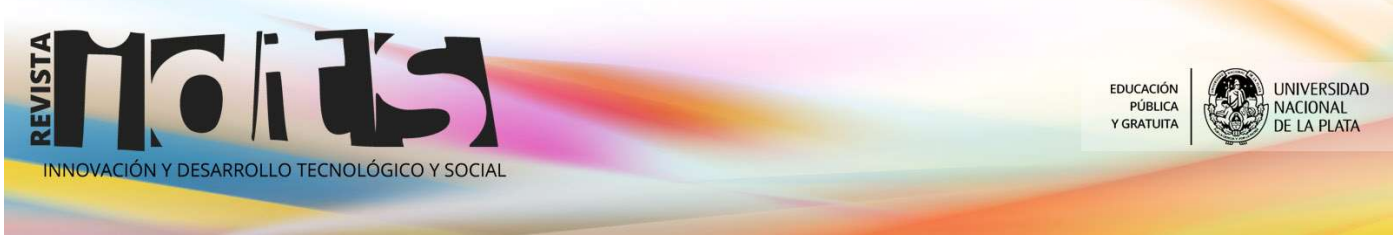

Innovación y Desarrollo Tecnológico y Social (2019) 1: 1-28

Los motores de tipo PMSM proporcionan una rotación de eje a una velocidad fija en sincronía con la frecuencia de la fuente de alimentación independientemente de la fluctuación de la carga mecánica -mayor o menor- que produce par resistente. La tensión (voltios) e intensidad (amperios) de la corriente descienden cuando actúa el Triac y de todos modos, el motor funciona a una velocidad de sincronismo; siempre que la frecuencia de la red sea constante, en este caso $50(\mathrm{~Hz})$; para cualquier par de torsión hasta el límite de funcionamiento del motor.

En el Sistema Internacional de Unidades (SI), la unidad del esfuerzo de torsión (también llamado: par motor) es la magnitud física: Newtons.metros (abreviado: N.m).

El par motor es el momento de una fuerza que se ejerce sobre el eje de transmisión de potencia (rotor). Según ciertos autores ${ }^{36}$, por fórmula de potencia de rotación, sabemos que: " $P=$. . $\omega$ " (Tipler-Mosca, 2006:265); donde cada símbolo algebraico significa:

$P$, es la potencia (medida en Watts).

T, es el par motor (medida en N.m).

$\omega$, es la velocidad angular (mediada en rad/s).

Si en ambas situaciones (Triac apagado y Triac en máximo ángulo de disparo), la velocidad angular $\omega$ (representada por omega), o velocidad de rotación medida en radianes/segundos (rad/s) es la misma: 314.159 (rad/s). Equivalente a las 3000 (RPM) obtenidas por la frecuencia de la corriente alterna de $50(\mathrm{~Hz})$.Despejando el par-motor o torque (tau): $\mathrm{T}=P / \omega$. Obtenemos los datos representado en la Tabla 2.

Esta es la clave del principio de funcionamiento y del ahorro del $59 \%$ del consumo de energía activa $(\mathrm{kWh})$, por reducción de la potencia activa $(\mathrm{kW})$. Según una análisis del ciclo de vida $(A C V)^{37}$ o LCA (en inglés) ${ }^{38}$, tal como lo describe la bibliografía del Ing. Guillermo Canale ${ }^{39}$ para el Posgrado de Ecodesing ${ }^{40}$ de la FBA-UNLP (Universidad

\footnotetext{
${ }^{36}$ Tipler, P. A.; Mosca, G. Física para la ciencia y la tecnología. 5ta. Edición. Barcelona: Editorial REVERTÉ, 2006 [En línea]. Disponible en: https://www.academia.edu/18965989/Fisica para la tecnologia [Accedido: 25-ene-2019]

${ }^{37}$ Canale, G. Et al. "Aportes de ACV simplificado al diseño para la sustentabilidad. Casos de aplicación industrial", en V Conferencia Internacional sobre Análisis de Ciclo de Vida - CILCA 2013 Mendoza: UTN, 2013 [En línea]. Disponible en: https://proyectaryproducir.com.ar/public html/Seminarios Posgrado/Bibliog obligat/CILCA\%202013\%20e n\%20castellano\%20FINAL\%2001-2013.pdf [Accedido: 25-ene-2019]

${ }^{38}$ En ingles: Life Cycle Assessment (LCA).

${ }^{39}$ Canale, G. Ciclo de Vida de Productos. Aportes para su uso en Diseño Industrial. Buenos Aires: INTI, 2013 [En línea]. Disponible en: https://proyectaryproducir.com.ar/wpcontent/uploads/2015/09/ACV\%20Libro\%20A4\%20Rev\%20b\%2016-12-13.pdf [Accedido: 25-ene-2019] ${ }^{40}$ Proyectar y Producir, equipo interdisciplinario de investigación en Diseño Industrial, "Programa para Seminario Taller de Posgrado FBA-UNLP. Diseño para la sustentabilidad social, económica y ambiental, 2016 [En línea]. Disponible en: https://www.proyectaryproducir.com.ar/?page id=305 [Accedido: 25-ene-2019]
} 
Nacional de La Plata), conforme a las normas internacionales ISO $14040^{41}$ e 14044; el D4S (diseño para la sostenibilidad) ${ }^{42}$ se centra en la quinta etapa del ciclo de vida: uso de la energía (eficiente). Siguiendo normativas como la adoptada en español UNE-EN ISO $14040^{43}$, a partir de la norma internacional ISO 14044.

Tabla 2. Para el primer caso, que el motor trabaja a máxima potencia, sin recorte de la onda AC (Triac apagado): $25.1(\mathrm{~W})$, los cálculos de la fórmula nos brinda un par-motor (torque) de: 25.1 (W)/314.159 $(\mathrm{rad} / \mathrm{s})=0.08(\mathrm{~N} . \mathrm{m})$. Para el segundo caso, que el motor trabaje a mínima potencia (con el Triac en máximo ángulo de disparo): $13(W)$, los cálculos de la fórmula nos arrojan un par motor (torque) de: 13 (W)/314.159 $(\mathrm{rad} / \mathrm{s})=0.04$ (N.m). Fuente: laboración propia. / Table 2. For the first case, that the motor works at maximum power, without trimming of the $A C$ wave (Triac off): $25.1(W)$, the calculations of the formula give us a torque (motor) of: $25.1(\mathrm{~W}) / 314.159(\mathrm{rad} / \mathrm{s})=0.08(\mathrm{Nm})$. For the second case, that the motor works at minimum power (with the Triac at maximum firing angle): $13(\mathrm{~W})$, the calculations of the formula give us a torque of: $13(\mathrm{~W}) / 314.159(\mathrm{rad} / \mathrm{s})=0.04(\mathrm{Nm})$. Source: self made.

\begin{tabular}{ccc}
\hline $\begin{array}{c}\text { Control de potencia } \\
\text { (Triac) }\end{array}$ & $\begin{array}{c}\text { Potencia activa } \\
\text { (Watts) }\end{array}$ & $\begin{array}{c}\text { Par-motor: torque } \\
\text { (Newton.metros) }\end{array}$ \\
\hline $\begin{array}{c}\text { Recorte de onda máximo } \\
\text { (Triac encendido en } \\
\text { disparo) }\end{array}$ & 25.1 & 0.08 \\
$\begin{array}{c}\text { Sin recorte de onda } \\
\text { (Triac apagado) }\end{array}$ & 13 & 0.04 \\
\hline
\end{tabular}

${ }^{41}$ ISO, Environmental management - Life cycle assessment - Principles and framework, ISO 14040, 15-jun1997 [On line]. Available: https://web.stanford.edu/class/cee214/Readings/ISOLCA.pdf [Accessed: 25-jan2019]

${ }^{42}$ TUDelf, DelfUniversity of Technology, DISEÑO PARA LA SOSTENIBILIDAD. Un enfoque práctico para economías en desarrollo. Países Bajos: TUDelf, 2007 [En línea]. Disponible en: http://www.d4sde.org/d4sspanishlow.pdf [Accedido: 25-ene-2019]

"43ISO, Gestión ambiental, Análisis del ciclo de vida, Principios y marco de referencia,ISO 14040:2006(es), 2006 [En línea]. Disponible en: https://www.iso.org/obp/ui\#iso:std:iso:14040:ed-2:v1:es [Accedido: 25ene-2019] 

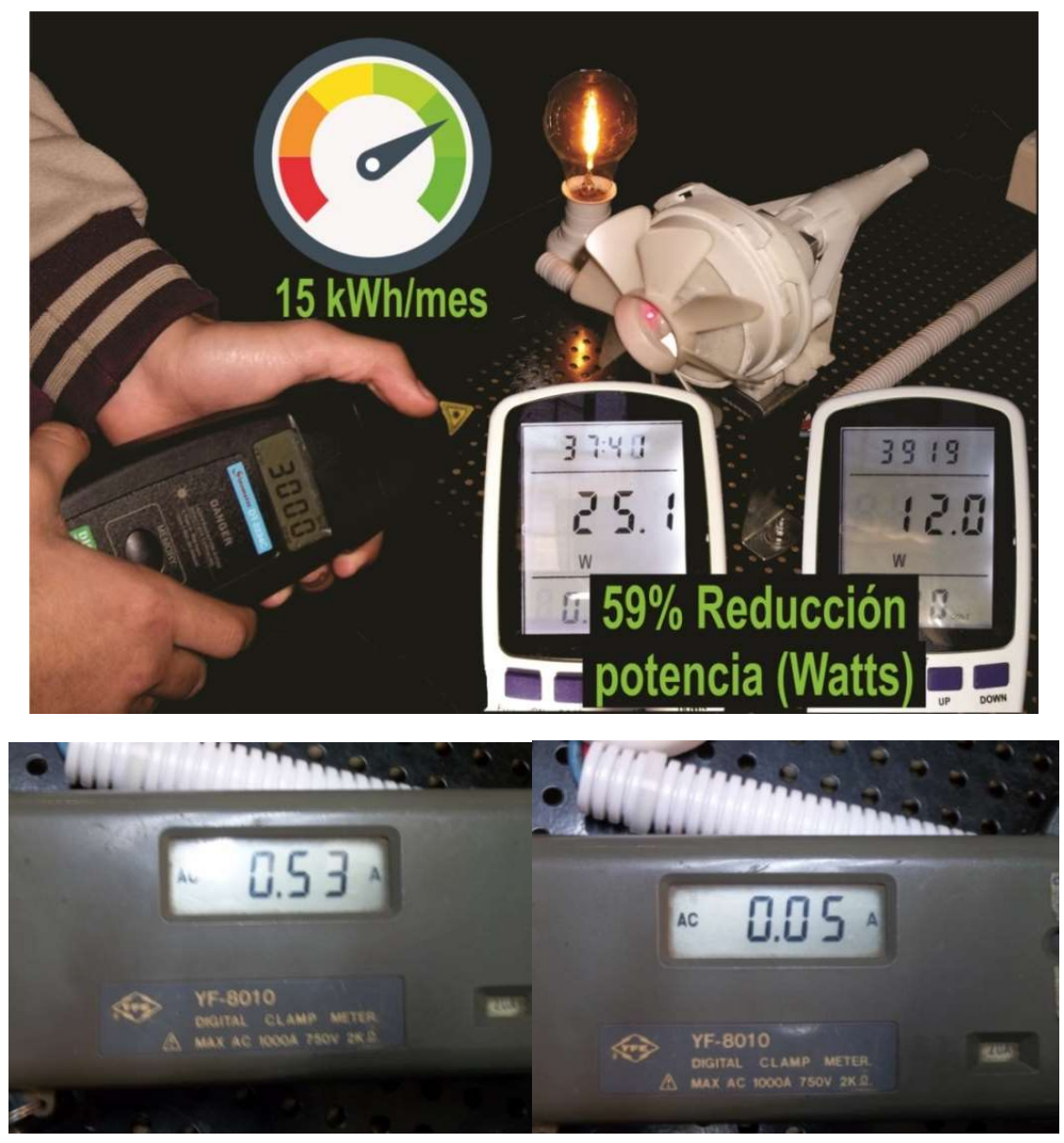

Figura $16(\boldsymbol{A}),(\boldsymbol{B})$ y (C).Cuando la eco-turbina trabaja a máxima potencia (Triac apagado), el vatímetro registra su valor mas elevado -25.1 (W)- y lapinza amperimétrica evidencia el mayor consumo -0.53 (A)-, siendo la velocidad angular verificada por el fototacómetro laser equivalente a 3000 (RPM). En el otro caso, cuando la eco-turbina trabaja a mínima potencia (Triac encendido en el mayor grado de disparo), el vatímetro registra su valor mas bajo -12 (W)- y la pinza amperimétrica evidencia el menor consumo -0.05 (A)-, siendo constante la velocidad angular verificada por el fototacómetro: 3000 (RPM). Valores TRUE RMS de potencia (vatios) e intensidad de la corriente eléctrica (amperios). Fuente: elaboración propia.Arriba foto tacómetro laser y vatímetro, abajo a la izquierza la pinza amperimétrica (True RMS) indica un consumo de 0.53 (Amperios) y abajo a la derecha la pinza amperimétrica indica un consumo de 0.05 (Amperios), lo cual significa una reducción de un 90\% de la intensidad de la corriente eléctrica. / Figure 16 (A), (B) and (C). When the eco-turbine works at maximum power (Triac off), the wattmeter registers its highest value $25.1(W)$ - and the clamp meter shows the highest consumption - $0.53(A)$-, being the angular velocity verified by the phototameter laser equivalent to 3000 (RPM). In the other case, when the eco-turbine works at minimum power (Triac on in the highest degree of trip), the wattmeter registers its lowest value $-12(W)$ - and the clamp meter shows the lowest consumption $-0.05(A)$-, being constant the angular speed verified by the photo-tachometer: 3000 (RPM). TRUE RMS values of power (watts) and current intensity (amps). Source: self made. Top photo laser tachometer and wattmeter, down to the left the clamp meter (True RMS) indicates a consumption of 0.53 (Amperes) and below to the right the clamp meter indicates a consumption of 0.05 (Amperes), which means a reduction of $90 \%$ of the intensity of the electric current. 


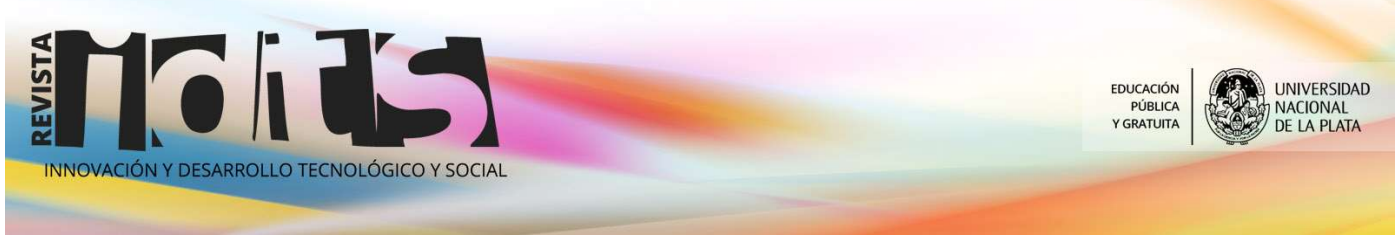

Innovación y Desarrollo Tecnológico y Social (2019) 1: 1-28

Información sobre el patentamiento/registro de la innovación o desarrollo:

El producto no está patentado, pero se puede patentar como invento y/o modelo de utilidad según corresponda, bajo la Ley de Patentes y Modelos de Utilidad $N^{\circ} 24.481$. Decreto 260/96 del texto ordenado de la Ley No 24.481, modificada por su similar No 24.572 (T.O. 1996) y su Reglamentación.

El prototipo no ha recibido financiamiento ni para su etapa de investigación y desarrollo $(\mathrm{I}+\mathrm{D})$, ni para su patentamiento.

\section{Agradecimientos}

Al Ing. Guillermo Canale y a la D.I. Rosario Bernatene que realizaron los esfuerzos necesarios para introducir el Posgrado de Eco Design en la carrera de Diseño Industrial en la Universidad Nacional de La Plata (UNLP), Provincia de Buenos Aires, República Argentina. También al D.I. Pablo Ungaro por poner a disposición el Laboratorio de Investigación del Departamento de Diseño Industrial (LIDDI-FBA-UNLP).

También al Ing. José Paramo de la carrera de Ingeniería Mecatrónica en la Universidad Nacional de Entre Ríos (UNER) y a los Ing. Marcelo Arlettaz, Carlos Blanc y Decano Jorge Penco de la Facultad de Ingeniería Eléctrica de la Universidad Tecnológica Nacional (UTN). Ambas Universidades -UNER y UTN- se encuentran situadas en la ciudad de Concordia, Provincia de Entre Ríos, República Argentina.

A todos ellos gracias sus enseñanzas académicas, sus comentarios, correcciones, sugerencias y haber puesto siempre en disponibilidad los laboratorios y equipos docentes de las universidades.

\section{Bibliografía}

Canale, G.; Bernatene, R.; Flores, F. (2013). APORTES DE ACV SIMPLIFICADO AL DISEÑO PARA LA SUSTENTABILIDAD. V Conferencia Internacional sobre Análisis de Ciclo de Vida -CILCA 2013-. Universidad Tecnológica Nacional. Mendoza. [On line]. Available: https://proyectaryproducir.com.ar/public html/Seminarios Posgrado/Bibliog obligat/CI LCA\%202013\%20en\%20castellano\%20FINAL\%2001-2013.pdf [Accessed: 25-jan2019]

Canale, G. (2013). Ciclo de Vida de Productos. Aportes para su uso en Diseño Industrial. Buenos Aires: INTI, 2013 [En línea]. Disponible en: https://proyectaryproducir.com.ar/wpcontent/uploads/2015/09/ACV\%20Libro\%20A4\%20Rev\%20b\%2016-12-13.pdf [Accedido: 25-ene-2019] 


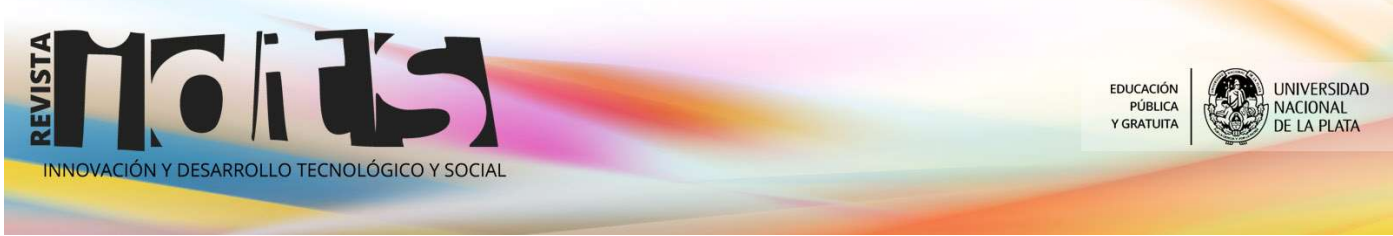

Innovación y Desarrollo Tecnológico y Social (2019) 1: 1-28

Canale, G. (2013). "Aportes de ACV simplificado al diseño para la sustentabilidad. Casos de aplicación industrial", en V Conferencia Internacional sobre Análisis de Ciclo de Vida - CILCA 2013 Mendoza: UTN, 2013 [En línea]. Disponible en: https://proyectaryproducir.com.ar/public html/Seminarios Posgrado/Bibliog obligat/CI LCA\%202013\%20en\%20castellano\%20FINAL\%2001-2013.pdf [Accedido: 25-ene2019]

Canale, G. (2014). Materialoteca. Perfil ambiental de materiales (solamente la Introducción). [En línea]. Disponible en: https://proyectaryproducir.com.ar/public html/Seminarios Posgrado/Bibliog obligat/Ex tracto\%20de\%20Introducci\%C3\%B3n\%20-\%20Materialoteca.pdf

[Accedido: 25-ene-2019]

Canale, G. (2010). S.O.S. Diseño sustentable. Sustentabilidad, Economía y Diseño. $5^{0}$ Foro de Ética y Sustentabilidad. Diseño Sustentable. Buenos Aires: 7/10/2009. Publicado en el Boletín No 158 del INTI. [En línea]. Disponible en: https://proyectaryproducir.com.ar/public html/Seminarios Posgrado/Bibliog obligat/IN TI\%20bol158-1\%20SOS\%20Dise\%C3\%B10\%20Sustentable.pdf [Accedido: 25-ene2019]

Catálogo digital, Concurso Nacional INNOVAR (2017). Ministerio de Ciencia, Tecnología e Innovación Productiva de la Nación. República Argentina [En línea]. Disponible en: https://mia.gob.ar/uploads/innovate/catalogo innovar 2017.pd [Accedido: 25-ene2019]

Harper, G. (2006). El ABC de las máquinas eléctricas III. Instalación y control de motores de corriente alterna. México: Grupo Noriega Editores. 355-370.

Instituto Argentino de Normalización y Certificación, Nuevas Normas IRAM de etiquetado de eficiencia energética, IRAM 62480:2017 [En línea]. Disponible en: http://aplicaciones.iram.org.ar/userfiles/files/medios/10-07/electrosector.pdf [Accedido: 25-ene-2019]

ISO, Gestión ambiental, Análisis del ciclo de vida, Principios y marco de referencia,ISO 14040:2006(es), $2006 \quad$ [En línea]. Disponible en:https://www.iso.org/obp/ui\#iso:std:iso:14040:ed-2:v1:es [Accedido: 25-ene-2019]

ISO, Environmental management - Life cycle assessment - Principles and framework, ISO 14040, 15-jun-1997 [On line]. Disponible en: https://web.stanford.edu/class/cee214/Readings/ISOLCA.pdf [Accedido: 25-ene-2019]

Ley 24481, República Argentina. Ministerio de Justicia y Derechos Humanos de la Nación. Presidencia de la Nación. InfoLEG: Información Legislativa [En línea]. Disponible en: 


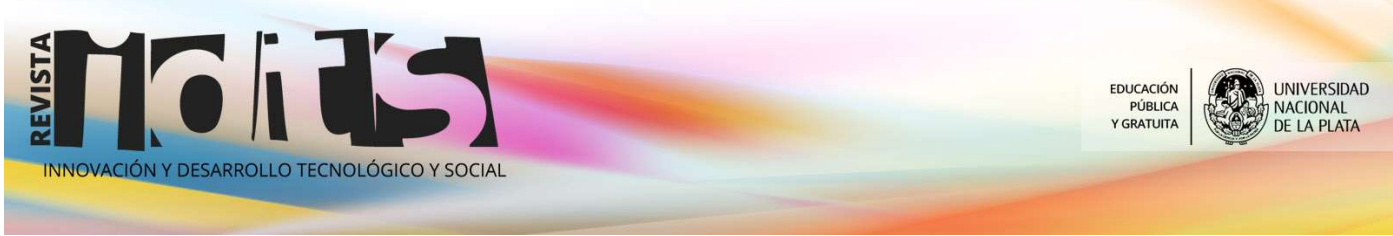

Innovación y Desarrollo Tecnológico y Social (2019) 1: 1-28

http://servicios.infoleg.gob.ar/infolegInternet/anexos/25000-29999/27289/norma.htm [Accedido: 25-ene-2019]

Ministerio de Justicia y Derechos Humanos de la república Argentina. Administración Pública nacional. Decreto 231/2015. Modificación del Decreto 357/2002. Buenos Aires [En línea]. Disponible en: http://servicios.infoleg.gob.ar/infolegInternet/anexos/255000259999/257246/norma.htm [Accedido: 25-ene-2019]

Nikola Tesla (1987), "Electro-magnetic motor", US 381968A [Online]. Disponible en: https://patents.google.com/patent/US381968A/en?oq=tesla+381968 [Accedido: 25ene-2019].

Proyectar y Producir, equipo interdisciplinario de investigación en Diseño Industrial, "Programa para Seminario Taller de Posgrado FBA-UNLP.Diseño para la sustentabilidad social, económica y ambiental, 2016 [En línea]. Disponible en: https://www.proyectaryproducir.com.ar/?page id=305 [Accedido: 25-ene-2019]

Sears; Zemansky. "31: Corriente alterna", Física Universitaria con Física Moderna, Volumen 2, México: Pearson Educación, 2009, pp. 1061-1091 [En línea]. Disponibleen: https://www.ucursos.cl/usuario/42103e5ee2ce7442a3921d69b0200c93/mi blog/r/Fisica General Fisica Universitaria Vol 2 ed 12\%28Sears-Zemansky\%29.pdf [Accedido: 25-ene2019]

Secretaría de Energía del Gobierno de la República Argentina. [En línea]. Disponible en: https://www.argentina.gob.ar/energia [Accedido: 25-ene-2019]

Secretaría de Energía del Gobierno de la República Argentina. Ahorro y eficiencia energética [En línea]. Disponible en: https://www.argentina.gob.ar/energia/ahorro-yeficiencia-energetica [Accedido: 25-ene-2019]

Sistema de gestión ambiental, ISO 14000 [En línea]. Disponible en: https://www.agroindustria.gob.ar/sitio/areas/d recursos humanos/concurso/normativa $\angle$ archivos/000007 Otras\%20normativas\%20especificas/000000 SISTEMA\%20DE\%20 GESTI\%C3\%93N\%20\%20AMBIENTAL\%20ISO\%201400.pdf [Accedido: 25-ene-2019]

Tipler, P. A.; Mosca, G. Física para la ciencia y la tecnología. 5ta. Edición. Barcelona: Editorial REVERTÉ, 2006 [En línea]. Disponible en:

https://www.academia.edu/18965989/Fisica para la tecnologia

[Accedido: 25-ene-2019]

TUDelf, DelfUniversity of Technology, DISEÑO PARA LA SOSTENIBILIDAD. Un enfoque práctico para economías en desarrollo. Países Bajos: TUDelf, 2007 [En línea]. Disponible en: http://www.d4s-de.org/d4sspanishlow.pdf [Accedido: 25-ene-2019]

Wildi, T. "17: motores síncronos", en Máquinas eléctricas y sistemas de potencia, México: Pearson Educación, 2007, p. 377-398 [En línea]. Disponible en: 


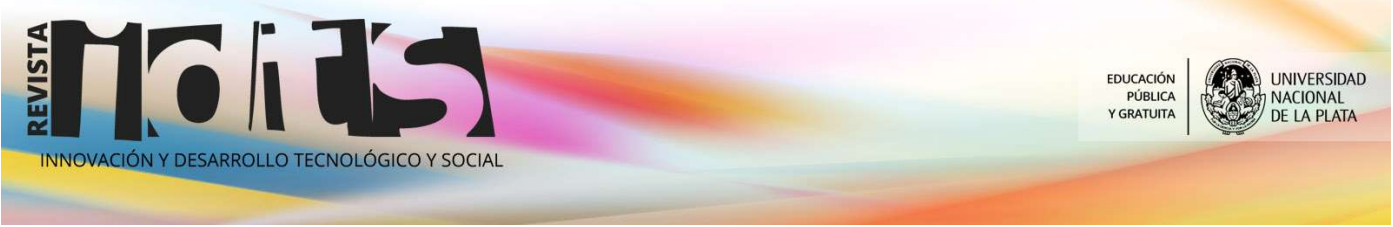

Innovación y Desarrollo Tecnológico y Social (2019) 1: 1-28

https://www.academia.edu/31911167/Maquinas Electricas y Sistemas de Potencia [Accedido: 25-ene-2019] 